\title{
Human Interaction With Robot Swarms: A Survey
}

\author{
Andreas Kolling, Member, IEEE, Phillip Walker, Student Member, IEEE, Nilanjan Chakraborty, Member, IEEE, \\ Katia Sycara, Fellow, IEEE, and Michael Lewis, Member, IEEE
}

\begin{abstract}
Recent advances in technology are delivering robots of reduced size and cost. A natural outgrowth of these advances are systems comprised of large numbers of robots that collaborate autonomously in diverse applications. Research on effective autonomous control of such systems, commonly called swarms, has increased dramatically in recent years and received attention from many domains, such as bioinspired robotics and control theory. These kinds of distributed systems present novel challenges for the effective integration of human supervisors, operators, and teammates that are only beginning to be addressed. This paper is the first survey of human-swarm interaction (HSI) and identifies the core concepts needed to design a human-swarm system. We first present the basics of swarm robotics. Then, we introduce HSI from the perspective of a human operator by discussing the cognitive complexity of solving tasks with swarm systems. Next, we introduce the interface between swarm and operator and identify challenges and solutions relating to human-swarm communication, state estimation and visualization, and human control of swarms. For the latter, we develop a taxonomy of control methods that enable operators to control swarms effectively. Finally, we synthesize the results to highlight remaining challenges, unanswered questions, and open problems for HSI, as well as how to address them in future works.
\end{abstract}

Index Terms-Human-robot interaction (HRI), human-swarm interaction (HSI), multi-robot systems, swarm robotics.

\section{INTRODUCTION}

$\mathbf{R}$ OBOT swarms consist of multiple robots that coordinate autonomously via local control laws based on the robot's current state and nearby environment, including neighboring robots. Key advantages of robotic swarms are robustness to failure of individual robots and scalability, both of which are due to the simple and distributed nature of their coordination. Multirobot systems that are not swarms have explicitly represented goals, form and execute both individual and group plans, have different capabilities, and can assume different roles [1][3]. Robots in these multirobot systems could act independently

Manuscript received March 6, 2015; revised June 27, 2015; accepted August 19, 2015. Date of publication October 15, 2015; date of current version January 20,2016 . This work was supported by the Office of Naval Research under Grant N00014-09-1-0680 and the European Research Council under grant PCIG14GA-2013-631553. This paper was recommended by Associate Editor A. M. Howard.

A. Kolling is with the Department of Automatic Control and Systems Engineering, University of Sheffield, Sheffield S10 2TN, U.K. (e-mail: a.kolling@ sheffield.ac.uk).

P. Walker and M. Lewis are with the Department of Information Science, University of Pittsburgh, Pittsburgh, PA 15260 USA (e-mail: pmwalk@gmail.com; ml@sis.pitt.edu).

N. Chakraborty is with the Department of Mechanical Engineering, Stony Brook University, Stony Brook, NY 11790 USA (e-mail: nilanjan.chakraborty@ stonybrook.edu).

K. Sycara is with the Robotics Institute, Carnegie Mellon University, Pittsburgh, PA 15213 USA (e-mail: katia@cmu.edu).

Digital Object Identifier 10.1109/THMS.2015.2480801 without coordinating, e.g., multiple robots searching a different area for victims in a search and rescue scenario. Conversely, they could also cooperate as a team in which all members work toward known shared goals, or coalitions in which members are self-interested. Swarms, on the other hand, involve coordination between robots that relies on distributed algorithms and information processing. Because of this, global behaviors are not explicitly stated and, instead, emerge from local interactions. In such cases, the individual robots themselves likely could not act independently in any successful manner.

Swarm robotics was originally studied in the context of biological swarms found in nature, but has since become its own distinctive engineering discipline [4]-[7], since it promises to be useful in a wide range of potential applications including reconnaissance, environmental monitoring, tracking, exploration, search and pursuit-evasion, infrastructure support, protection, and even space exploration [8]. Despite their potential, most robot swarms are still confined to laboratory settings and simulations. There are a variety of robot simulation platforms that have been used for studies and benchmarking, such as the widely used Stage platform [9], which offers 2-D simulations that scale to thousands of robots.

A number of recent projects have made some progress developing swarm hardware. The Micro Autonomous Systems and Technology project has created numerous microvehicles [10]. The "Swarmanoid" Towards Humanoid Robotic Swarms" project [11] developed a swarm of heterogeneous mid-sized robots, including the popular SWARM-BOT platform s-bot [12]-[14]. Other projects and experiments used available platforms including the Kobot [15], E-puck, and Kilobot [16], [17]. These examples, along with growing development of robotic hardware and its decreasing cost suggest that real-world applications for swarms are within reach. To achieve this, a number of challenges remain to be addressed-primarily, the study of human interaction with such swarms. For the most part, swarms are expected to operate autonomously. However, the presence of a human operator can be beneficial and even necessary since the operator could: 1) recognize and mitigate shortcomings of the autonomy; 2) have available "out-of-band" information not accessible to the autonomy and that can be utilized to increase performance; and 3) convey changes in intent as mission goals change.

There is currently a dearth of studies investigating effective ways in which human supervisory control of swarms could be performed. This paper is an attempt to fill this gap by outlining the basic concepts, requirements, and challenges of humanswarm interaction (HSI), and by reviewing related literature within this emerging field to identify issues and important areas for further work. 
In the following, we will first briefly discuss swarm robotics in Section II. This will set the context and provides the uninitiated reader a cursory glance on this growing field. Then, in Section III, we will discuss HSI from an operator perspective. Section III-A establishes the operator's perspective by introducing cognitive complexity and a notion of difficulty for the control of large swarms. Within this context, we address the following research questions.

1) How do the properties of the communication channel between operator and swarm affect HSIs, such as the ability to observe and control the swarm?

2) How can an operator observe a swarm and its dynamics?

3) What are the different control methods used, and how do they affect the ability of an operator to control a swarm?

4) What is the relevance of the notion of levels of automation in HSI and how has it been exploited and studied?

5) How do swarm dynamics affect the ability of the operator to control the swarm?

Question 1 is addressed in Section III-B by discussing issues of operator-swarm communication. This is followed by Section III-C in which we address question 2 by discussing swarm state estimation and visualization. Then, in Section III-D, we develop a taxonomy for methods of control with which an operator can impart intent to the swarm, thereby addressing question 3. Question 4, relating to levels of automation, is addressed in Section III-E. Question 5 is addressed in Section III-F with an emphasis on the timing of operator inputs. Finally, we conclude with a discussion in Section IV and present suggestions for further work in Section V.

\section{ROBOT SWARMS}

In one of the first surveys discussing swarms, Dudek et al. [18] propose a taxonomy that emphasizes tasks. They distinguish between tasks that 1 ) require multiple agents, 2) are traditionally multiagent, 3) are inherently single-agent, and 4) may benefit from the use of multiple agents. For the latter types of tasks, using a multirobot system has to be justified with respect to some performance criteria. These criteria are usually expressed in terms of efficiency, effectiveness, robustness, flexibility, or design complexity. Tasks corresponding to 1) or 2) are frequently mentioned in other surveys [1], [2], [19]-[21] and are most often spatially distributed tasks. In addition to tasks, a taxonomy based on system properties is also found in [18] which classifies systems according to 1) size, 2) communication range, 3) communication topology, 4) communication bandwidth, 5) reconfigurability 6) processing capability of each unit, and 7) composition. A clear distinction between swarms and multirobot systems is not made. In fact, earlier versions of [18] used these terms interchangeably.

Other taxonomies, such as [2], [19], [20], and [21], distinguish multirobot systems not based on hardware features but rather on problems, solutions, issues, and research areas. In [19], Cao et al. distinguish between systems based on group architecture, resource conflicts, origins of cooperation, learning, and geometric problems. Parker, in [2], focuses on different approaches to designing a distributed intelligence, namely the bioinspired paradigm, the organizational and social paradigms, and the knowledge-based, ontological, and semantic paradigms. Similarly, and also focused on coordination, in [1], Farinelli et al. propose a taxonomy of multirobot systems that distinguishes whether robots are aware or unaware of each other.

An emphasis on swarm systems, rather than more general multirobot systems, is found in [5], which focuses on the commonly desired swarm properties of robustness, scalability, and flexibility. In [6], Brambilla et al. propose two taxonomies: one classifying methods for design and analysis of swarms and the other classifying types of swarm behaviors. Another recent survey [7] also includes a list of recent projects and descriptions of physical robots, projects, and simulation platforms.

From this vast trove of taxonomies and descriptions of multirobot and swarm systems, we will present selected examples and problems to give a brief introduction to swarm robotics as a whole. We will not rely on a specific taxonomy, but rather discuss swarm systems from the perspective of different methodologies, selected tasks, and algorithms that one may run on a swarm in practice.

\section{A. Swarm Models}

Swarms have been studied from a number of perspectives, including bioinspired, control theoretic, amorphous computing, and physics-inspired. The models and methods that originated from these differ not only with regard to the source of inspiration but also with regard to theoretical guarantees, operating conditions, and suitable metaphors. The latter may have some bearing with regard to the interpretation of a swarm behavior by human operators. Thus, it is necessary to understand these commonly used swarm models if one is to design a human-swarm system around them.

1) Bioinspired: Biological systems have long since been an inspiration for the design of robotic systems in terms of hardware [22] as well as behavior [23]. Much of the work on swarm robotics originated from the study of biological swarms and swarm intelligence [4]. A recent survey [6] reviewed swarm engineering efforts and identified four areas that require further attention to support the development of real-world applications, namely 1) modeling and specification of requirements, 2) design of swarm behaviors, 3) verification and validation of swarm models, and 4) HSI. The most interesting for the perspective of this paper is the fourth area, concerned with operation and maintenance of swarms. In this area, particular concern is given to enabling effective control when lacking a centralized instance.

One of the better known examples of a swarm algorithm derived from a biological inspiration is presented in [24]. Therein, Couzin et al. model the spatial behavior of animal groups with simple local interaction rules. These rules are determined by three parameters, the radii of three zones, namely zones of repulsion, orientation, and attraction. In the paper above, this simple model can generate four qualitatively distinct swarm behaviors: 1) swarm; 2) torus; 3) dynamic parallel; and 4) highly parallel. Which of the resulting behaviors a swarm generates depends on the choice of parameters and initial conditions and raises the obvious question on how a human operator could interact with such a biological swarm model to induce transitions between 
these four types or change the direction of motion for a given type. This question has been investigated in [25] through the injection of leaders and predators under the control of an operator, a paradigm that will be discussed further in Section III-D4.

Another strand of bioinspired research is related to pheromone-based communication [26], [27]. Pheromones have been used in [28] to coordinate a swarm to for surveillance, reconnaissance, hazard detection, and path finding. On a more general note, in [29], Sumpter identifies several principles that describe biological systems that exhibit collective behavior. Applying these principles to engineered systems has led to a wide range of bioinspired systems; some of which are surveyed in [30].

2) Control Theory: There has been a considerable amount of work done on swarms from the perspective of control theory-a brief survey of which is found in [31]. Some of this work has been done under the heading of distributed robot networks [32]. The authors of [32] unify prior work on connectivity maintenance [33], rendezvous [34], deployment [35], [36], boundary estimation [37], and tracking [38], and present a rigorous model for control and communication in such networks. The physical model of individual robots is defined in [32] as a continuoustime continuous-space dynamical system with a state space, input space, allowable initial states, and a control vector field that determines robot motion given a state and input. The network aspects are modeled as a communication edge map, which determines whether a communication link between any two robots exists. This is followed by a formal definition of control and communication laws, with discrete-time communication but continuous-time motion.

The practical advantage of this approach is the generalized consideration of physical dynamics, which have received less attention in bioinspired work. While the formal results are important, their underlying assumptions are necessarily simplified to make them tractable. Yet, resulting formal guarantees and analysis tools could still be useful for human operators and system designers. For instance, in [39], formal methods are used to determine whether human control inputs for certain swarm tasks are theoretically possible. Control-theoretic approaches are, therefore, an important complementary contribution to bioinspired works.

3) Amorphous Computing: Amorphous computing [40] refers to the programming of many small computers distributed irregularly throughout some surface or volume, with no a priori knowledge of their location or neighbors [41]. These small computers are each controlled through identical programs, which dictate their behavior through interactions with nearby nodes. These computers form a discrete approximation of the continuous space they inhabit and, thus, can be controlled programmatically through gradients or vector fields. The amorphous computing idea is thus strikingly similar to swarm robotics in general. Amorphous computing assumes few capabilities of the individual units-typically only an on-board clock, some method of short-range communication, a power source, and the sensors and actuators necessary for their application. The setup is also robust to communication failure or failure of a unit as a whole, because mechanical failure simply means one less point with which to estimate the continuous medium.

A programming language Proto was developed to deal with distributed computers in a medium, and to determine the specific engineering problems that need to be solved before real-world applications of swarms operating under the amorphous abstraction can come to fruition [42]. Proto allows an operator to compose behavioral primitives for their swarm. The authors of [43] and [44] have used Proto to create an amorphous computing system comprised of about 10000 individual robots and a realworld system of 40 robots where they tested swarm behaviors. Tests using the real robots indicate that the system is relatively robust to communication message drops and lag times, and that swarms programmed under amorphous computing can successfully demonstrate simple swarm behaviors, such as rendezvous and dispersion.

4) Physics-Inspired: Physical systems are yet another important source for algorithms with emergent properties. A wellknown example is [45], where the authors present a system of self-propelling particles that achieve alignment following simple update rules. Subsequently, Jadbabaie et al. [46] provide a rigorous formal analysis of such types of systems from a control- and graph-theoretic perspective. The neighbor-based rules therein for coordinating the motion of particles are not unlike some flocking algorithms inspired by biological systems. In [47], also inspired by artificial forces, an inverse-power law is used to determine attraction and repulsion forces between robots and groups of robots, coined social potential fields. Another example of using a force-based law is found in [48], which also includes obstacles in the force equations. Yet another approach that seeks inspiration from the natural world is known as physicomimetics [49]-[51]. The key idea here is that physics in and of itself is a discipline that describes large-scale emergent phenomena in terms of well-understood equations, but which arise from a multitude of lower level interactions (of particles and forces). The approach has been applied in [52] and [53].

Despite the similarities to bioinspired approaches for flocking, the physics-inspired work has a distinctly different perspective on the individuals in a swarm. The focus is more on passive than active interactions with a different perspective on agency (e.g., particles do not communicate actively and only influence each other tacitly through forces). One of the main advantages of a physics-inspired approach is the considerable body of experimental and formal work relating to self-organization in physical systems that one can borrow from. For example, work on predictive self-assembly [54] of polyhedra has been useful for determining how to generate self-assembled structures, i.e., in [55], it was shown how to generate a self-assembled structure by setting desired nearest neighbor distances. In a swarm, this could be expressed by having each member move to a position that most closely achieves the desired interrobot distances.

\section{B. Swarm Tasks and Behaviors}

Existing surveys on swarm robotics provide an excellent and detailed overview of the large number of swarm behaviors that 
have been studied, most of which solve a specific task. Some include categories for these behaviors, such as in [6], which distinguishes spatially organizing, navigation, and collective decision-making behaviors. In the following, we will present a few selected examples.

1) Aggregation and Rendezvous: One of the simplest swarm behaviors is aggregation, a process often found in natural swarm systems [56] and adapted to artificial swarms (see, for example, [57]). From a control-theoretic perspective, a similar problem has been studied as the rendezvous problem [34]. The basic objective for both is to move all swarm robots toward a common location.

Bioinspired aggregation behaviors have been implemented on real swarm robots in [57]. Therein, the authors start with a model for a specific swarm robot, the s-bot, equipped with an omnidirectional speaker, three-directional microphones, and eight infrared proximity sensors. Weights for a neural network controller, with direct connections from every sensor to every actuator, are evolved under a fitness function that measures aggregation via the average distance of robots from the center of mass of the swarm. Two distinct aggregation behaviors were discussed: one leads to multiple static aggregates, while the second leads to a single moving dynamic aggregate that resembles a flocking behavior.

The rendezvous problem has been studied in [34]. Therein, the authors define an abstract model of a robot that knows its own location and can transmit it to neighbors within its communication network. The authors prove theoretical guarantees for the convergence of the swarm to the circumcenter under different static and changing communication topologies. The main assumptions for guarantees to hold are the ability to sense or receive the locations of neighboring robots and having an environment without obstacles. Further work on the rendezvous problem has led to a reduction in the required sensor capabilities. For example, in [58], Yu et al. present a solution to the rendezvous problem that does not require knowledge about exact location of other robots, but instead uses only a quantized bearing sensor that reports the presence of another robot in a small range ahead of the robot.

2) Deployment and Area Coverage: Deployment of swarms, i.e., swarm dispersion governed by local control laws, is a swarm behavior typically used for area coverage. Swarms are expected to be ideal for area coverage, because this task requires covering, with sensors, a large area in order to observe some phenomena of interest or discover and track targets. One of the first to apply a force metaphor (a physics-inspired perspective) for the distribution of large robot teams are Howard et al. in [48]. Therein, robots are repelled by obstacles and other robots and, as a consequence, distribute throughout an environment with obstacles. Experiments with 100 robots show successful dispersion in a realistic office environment and convergence to a static equilibrium.

A different approach to area coverage, with the goal of seeing every part of an environment, akin to the art gallery problem, is taken in [36]. Therein, the environment is given by a polygonal boundary and robots cover the environment by creating an incremental partition of the environment as they progress to cover it. Some results regarding convergence time and guarantees for a given number of robots are provided. A fleet of 56 real robots was used in [59] to test and compare five area coverage algorithms showing significant differences between the time to reach various goal locations and to fully disperse in the entire environment.

3) Flocking and Formation Control: A more complex set of swarm behaviors is the formation of specific patterns of motions, specifically flocking, or consensus on a direction and speed of movement. One of the first algorithms to enable a swarm of robots to flock was presented by Reynolds in [60], with the motivation to simulate flocks of birds for computer graphics. Therein, individuals would follow simple local rules to avoid collisions (separation), match velocities to their neighbors (alignment), and center themselves among their neighbors (cohesion). Together these generate a flocking behavior. One of the earlier demonstrations of how to control a flock of animals, with robots influencing the flock, was presented in [61]. A simple controller for the robot was tested in a simulation with a swarm model similar to [60]. In [62], work on flocking is applied and implemented on robots with particular emphasis on the translation of control inputs to robot motion. More precisely, the force vectors resulting from the flocking rules for cohesion, separation, and alignment are translated into forward and angular velocity. The experiments in [62] show improved effective travel distance when considering magnitudes of the forces.

An overall framework for the analysis of flocking algorithms, including analysis of swarm fragmentation, is presented in [63] (following a line of work from [46], [64], and [65]). One of the most interesting aspects of [63] is the first introduction of a formal definition of what constitutes flocking. This definition is established with regard to 1) how much the flock differs from a lattice (i.e., a formation with all neighbors having a desired distance to each other) in terms of a deviation energy, 2) to what extent velocities are matched, and 3) connectedness and cohesiveness of the flock.

4) Foraging and Transport: Formation of chains between two locations, akin to ant trails, constitute a more complex behavior [66]. The key challenge for the chain formation is to establish shortest paths that can also be used by a larger number of swarm robots without leading to congestion. Other works have dealt with cooperatively transporting a single object with multiple robots [67]. An overview of a range of the work done on this problem is found in [68]. A bioinspired perspective for foraging is given in [69], whereby a stigmergy-based approach, inspired by the pheromone markers of ants, is presented for a heterogeneous swarm composed of ground and aerial robots.

\section{HUMAN-SWARM INTERACTION}

In this section, we present the key components of a humanswarm system while focusing on the perspective of the operator. These are illustrated in Fig. 1. We begin in Section III-A by discussing general issues of cognitive complexity when interacting and completing tasks with swarms. The operator interacts with the swarm through an interface that is constrained by the means of communication and relies on methods for state estimation and 


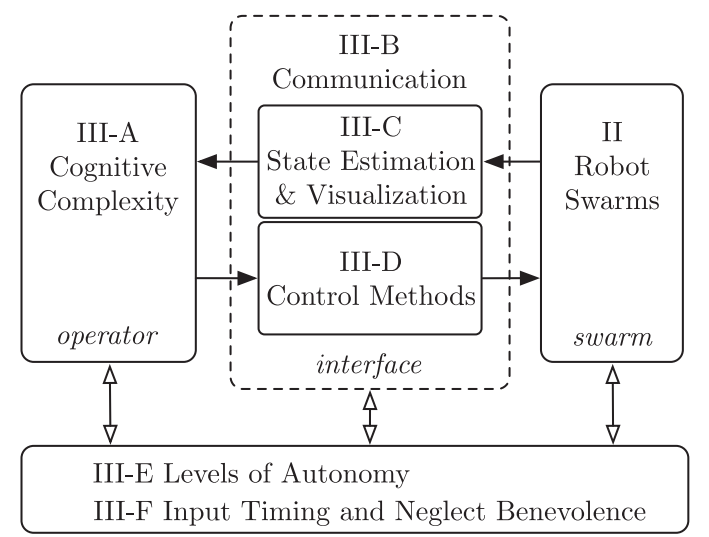

Fig. 1. Key components of a human-swarm system, with an operator solving complex tasks and communicating with a swarm through an interface to receive state feedback and send inputs using appropriate control methods. The entire system is influenced by levels of automation and input timing and neglect benevolence. Section indices show our organization.

visualization and control that facilitate the interaction between human and swarm. Communication is discussed in Section III$\mathrm{B}$, followed by state estimation and visualization in Section III-C. Subsequently, we discuss different methods with which the operator can control a swarm in the form of a brief taxonomy in Section III-D. Issues regarding levels of automation as well as input timing and neglect benevolence, which influence the overall human-swarm system, are discussed in Sections III-E and III-F, respectively.

\section{A. Cognitive Complexity of Human-Robot Systems}

Earlier taxonomies of multirobot systems have focused primarily on physical characteristics, tasks, and methods, while human-robot interaction (HRI) taxonomies have considered roles and structure. Few, however, have addressed the difficulty of the operator's tasks. In computer science, the notion of computational complexity - the time that must be used to solve a problem as a function of the size of its input-has proven fruitful for separating scalable and tractable algorithms from nonscalable ones. Algorithms with high complexity may work for small problems, but fail or grow inefficient for even slightly larger ones. The task of controlling multiple robots is similar to an algorithm in that the operator must perform a repetitive sequence of decisions and actions to enable the system to reach some desired goal state.

In [3] and [70], HRI was defined in terms of operator's cognitive effort akin to computational complexity. If a group of homogeneous robots are performing independent activities, the operator can devote the same attention to each in turn, resulting in a complexity of order $n$, written $O(n)$, because each of the $n$ robots requires the same set of operator interaction with it. Thus, the total operator effort/attention is linearly related to the number of robots. Applications of this $O(n)$ interaction complexity are search and rescue when the area has been divided in regions that are searched by robots operating independently of one another, and authentication of weapons release where the operator must authenticate each release sequentially, etc. A benefit of this independence is that more robots can be controlled

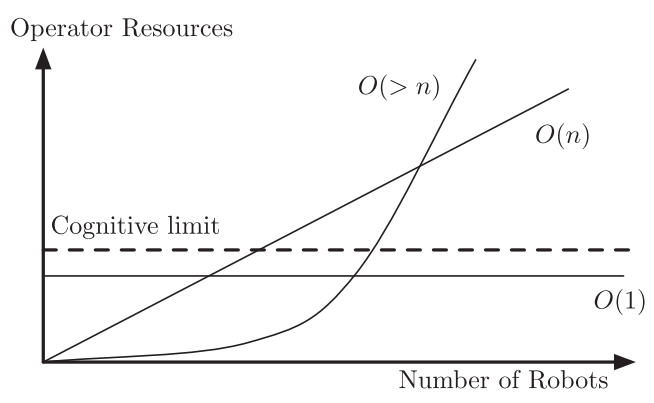

Fig. 2. Graphical illustration of the concept of control complexity in a humanmultirobot system.

simply by adding more operators in a linear manner. Indeed, the fan-out model proposed in [71] to estimate the number of robots an operator can control within some time interval is a special case of the cognitive complexity of control scheme proposed by Lewis [3], [70]. The fan-out model makes the assumption of Neglect Tolerance, namely that a robot's performance will degrade if the robot is left unattended by the operator for some time (neglect time) and that some interaction time must be periodically devoted to the robot by the operator. More sophisticated formal schemes for scheduling operator attention have been recently developed [72], [73] as well as human studies to determine operator behavior under those scheduling schemes [74]-[76].

A different form of control, such as designating a region to be searched by drawing it on a map, can command an arbitrary number of robots with a single operator action, as long as the interactions between the robots (such as preventing collisions) can be handled autonomously. In this case, the number of actions the operator must take are independent of the number of robots, and thus, control is $O(1)$, allowing one (or a fixed number of) human operator(s) to control any number of robots. Given a robotic swarm where the members are coordinating autonomously to provide useful behaviors, such as flocking and rendezvous, control of the swarm can be $O(1)$, thus making swarms a desirable multirobot organizational scheme, where the operator need only focus on the goal of the swarm overall. This, in effect, means that the operator can treat the swarm as a single entity much of the time, and multiple robots can be added or removed without impacting the cognitive burden of the human operator. However, in cases where the operator must divide the swarm, or issue separate commands to different subswarms, control complexity may more realistically lie between $O(1)$ and $O(n)$, or potentially worse.

In contrast with the above two scenarios, there also exist tasks where robot-to-robot interaction is not handled autonomously; yet, the robots must coordinate to perform some common task, such as box pushing with robots controlled by an operator [77]. Such a scenario would have superlinear command complexity, $O(>n)$, because dependences between robots create cascading demands as the number of robots grows. See Fig. 2 for a graphical illustration of these concepts.

The primary purpose of the cognitive complexity scheme is to emphasize the effort of the human operator required to control a multirobot system, and as such, the basic notion is applicable 
to swarms as well, and contextualizes HSI. The notion and scheme of cognitive complexity is useful in HSI in that it can be used to guide development of algorithms that remove the necessity to manage interdependences between robots in the swarm. The overall cognitive difficulty for swarm control is, however, also determined by the parts of the control loop detailed in the following sections and is not always $O(1)$.

\section{B. Communication}

The majority of research on HSI has focused on remote interactions (i.e., when the human operates separately from outside the swarm). For such interactions, the dominating issue is that of communication, usually with an operator at a computer terminal. Communication is also one of the main challenges in swarm robotics in general, in particular with regard to the topology of the swarm network. As briefly noted in Section II, most proofs of guarantees for swarm behaviors have to carefully take into account changes in the communication topology, as these are influenced by robot motion, which in turn depends on inputs that may change when the topology changes. The difficulty here lies primarily in guaranteeing certain properties of the evolution of the communication topology that hold regardless of how they influence swarm motion. Fragmentation of a swarm into multiple connected components is a particular concern. A human operator will likely have to account for these communication difficulties as well. In addition, a remote swarm operator needs remote access to relevant information about the swarm, a problem that an autonomous distributed control algorithm does not face since it runs directly on the robots. Some challenges regarding communicating this information to an operator and the effect of resulting uncertainty from incomplete information are briefly discussed in [78].

Proximal interactions, on the other hand, assume that operators and swarms are in shared environment. Such interactions are suitable to support local interactions between swarms and operators and generally do not require a communication infrastructure. Multiple operators can easily be distributed across the swarm and environment. Some swarm robotics surveys that discuss the need for HSI research [5], [6] desire such a local interaction scheme in order not to interfere with the distributed design of swarms. In the following, we discuss communication issues related to remote and proximal interaction schemes.

1) Remote Interaction: Despite the difficulties mentioned above, remote interaction is likely to be the default option for swarms that are entering otherwise inaccessible or dangerous areas. In fact, one of the key motivations for using swarms in real-world applications is their ability to be deployed in exactly such areas. Hence, one of the primary challenges of HSI is to reconcile the distributed nature of swarms with a central human element of control and the ability to collect information about the swarm and environment. Part of this is a technical challenge, addressed in the study of sensor networks [79], [80] and mobile ad hoc networks [81], [82]. It is noteworthy that swarm methods and algorithms are also used to manage networks, e.g., they are used in [83] to improve bandwidth and latency and in [84] to design routing protocols.
There may still be individual robots that are capable of global communication with an operator. An operator might also be able to broadcast a command to an entire swarm. Therefore, we can have global one-to-one or global one-directional one-to-many communication. For example, underwater gliders that resurface to establish a brief satellite connection and then return to the swarm enable one-to-one global communication.

An example of a distributed swarm network that is controlled by a central operator is found in [85]. Therein, the authors address a number of practical challenges for maintaining a swarm with 112 robots. A so-called gateway robot receives new software and broadcasts it into the swarm to enable the programming of these robots. A centralized user interface allows an operator to receive data from the gateway robot about the swarm state.

The important practical problems facing a swarm operator are latency, bandwidth, and asynchrony. From the existing swarm literature, one can draw the conclusion that for swarm systems, bandwidth is more limited and latency and asynchrony higher than in other types of systems. There are few experiments regarding the impact of bandwidth limitations on HSI although. One first attempt was made in [86] by exploring three bandwidth conditions in a foraging task. In the low-bandwidth condition, the operator only receives a location update from a single robot per time step. In the medium-bandwidth condition, the swarm utilizes local bandwidth to estimate the swarm centroid and average orientation, which is then transmitted to the operator. In the high-bandwidth condition, all swarm robots communicated their location to the operator at every time step. The performance of operators in the medium- and high-bandwidth conditions was statistically indistinguishable, suggesting that not all position data from each robot in a moving swarm are necessary for proper control. The effect of latency on human control of a foraging swarm was investigated in [87]. Increase in latency was associated with deteriorating performance; however, a predictive display that took into account swarm dynamics helped to lessen the negative effects of latency.

2) Proximal Interaction: Proximal interactions with a swarm enable an operator to observe the whole or part of a swarm directly and interact in a shared environment. In cases when the swarm can sense the operator, the latter can act as a special swarm member and thereby influence the behavior of the swarm through local interactions. This also opens the possibility for having multiple human operators who can influence and control the swarm in a distributed manner.

Most of the research on proximal swarm interactions has focused on enabling the interaction through gesture recognition [88]-[90] as well as face engagement and speech [91]. The distributed gesture recognition presented in [88] and [90] facilitates the communication of a wide range of instructions to all swarm robots within sight. The human wears an orange glove that is easily recognizable by the cameras on board the robots. The robots that can see the glove then participate in a consensus protocol to determine the meaning of the gesture. Line of sight is also required for the face engagement and speech approach used in [91]. Therein, the operator can select one or multiple robots via face engagement, which is detected via a camera on each robot, and speech commands. With speech commands, the 
operator can add or remove engaged robots to a group or trigger a desired behavior. Both mechanisms would, in theory, enable the integration of multiple operators into a swarm, although such experiments have not been carried out yet. Proximal interactions were envisioned in the GUARDIANS project [92] as beneficial for firefighters in a rescue scenario, and in [93], the human operator interacted with the swarm as a special swarm member that acted as an attractor.

Proximal interactions with a swarm that actively engage an operator, such as speech or gestures, are similar to proximal interactions with other robot systems [94] or interactions in the context of peer-to-peer teaming [95]. The added difficulty for swarms results primarily from limited sensing and computational power on individual robots. Distributed methods may mitigate this shortcoming and additionally benefit from multiple sensor estimates (e.g., multiple perspectives for cameras). Proximal interactions that treat the operator as an ordinary or special swarm member are usually not found in other human-robot systems. However, such passive proximal interactions have received little to no attention in the literature so far, and it is not clear how one would utilize them for controlling large swarms.

\section{Swarm State Estimation and Visualization}

Proper supervision of a semiautonomous swarm requires the human operator to be able to observe the state and motion of the swarm, as well as predict its future state to within some reasonable accuracy. How good the prediction must be depends on the scenario, but there must be some ability to forecast future behavior in order to relate to the effects of control inputs. A key distinction between swarms and multirobot systems is a focus on the swarm as a single entity rather than multiple individual robots.

An important function of the human operator is to estimate the state of the swarm over time so as to be able to provide appropriate control inputs. The main difficulty here is not only to visualize the swarm state but also to facilitate the understanding of swarm dynamics as well as the impact of control inputs. The swarm models, i.e., bioinspired, control-theoretic, amorphous computing, and physics-inspired models, may offer suitable metaphors for this problem. For example, a visualization of forces might aid comprehension for an operator familiar with attractive and repulsive forces. Very little research, however, has investigated these ideas.

State visualization is particularly difficult for the operator in situations with incomplete information. Such situations arise in the real world from constraints on bandwidth and communication latency that arise in operations taking place in remote locations as well as sensing errors and uncertainty. Several recent studies explored how different types of displays could help the operator effectively visualize the state of the swarm. In [86], the authors show that when information is restricted to just the swarm centroid and standard deviation of positions, human performance on a target search and navigation task was unhindered, despite localization errors of individual robots. Similarly, in [87], the authors focus on latency in the communication channel between the swarm and human. This also mimics sim- ilar scenarios to the bandwidth case, where a human operator may be controlling a swarm that is far away, or in an environment difficult for radio waves to penetrate. Here, the authors found that even a simple predictive display was beneficial to operators performing a target-searching task. These early studies indicate that simplifying the large state of a swarm to a lower dimensional representation can be beneficial to control. Other researchers [96] have shown that small samples of angular velocities and concentration of neighbors can be sufficient to classify the behavior of a swarm following a common flocking algorithm [97] as either flocking (moving in a common direction) or torus (moving in a circle). Reducing the amount of noise and aggregating and fusing information to simplify the problem of determining a swarm's state are promising research areas.

Besides displays, multimodal feedback to the operator has also been investigated [98]. Here, the authors used a potential field approach for controlling the swarm for a convoy protection scenario and designed an interface that provides feedback regarding the swarm speed, strength, capability, and dispersion. The feedback was presented as visual, auditory, and tactile or a combination thereof. A study with 16 participants was carried out in which operators had to respond to swarm feedback with lower response times in the multimodal feedback conditions.

Besides the aspect of designing appropriate algorithms that provide aids to humans for swarm state estimation, there is a very important issue of whether humans may be able to learn to understand swarm dynamics, given appropriate feedback. This question has hardly been investigated and is essential for operators that wish to change or properly assess swarm behavior. In [99], the authors investigate whether human operators can learn to predict the effects of different input behaviors to a simulated swarm. The authors use a two-choice control task, whereby operators choose either a dispersion or a rendezvous algorithm for a swarm randomly distributed in an environment with obstacles. The goal was to cover as much of the environment as possible in each trial. Results from the experiments showed that human performance increased over the 50 trials from an average of $60 \%$ to $80 \%$ correct, thus indicating that humans could learn to estimate the results of deploying a particular behavior on task performance. The results of this study are interesting from another perspective as well, because they were used to create a computational cognitive model of the human operator that mimicked the human performance [100]. To our knowledge, this is the only study using a cognitive architecture to model human operators in an HSI task.

In [101], the authors investigate whether human operators can acquire enough understanding of swarm dynamics to estimate the effects of the timing of their control input. In this study, operators were tasked with observing a swarm moving from a random initial state to some first formation, and determining the optimal time to give an input signaling the swarm to move to a second, goal formation. The operators had to give the input at the time that would minimize the convergence time to the second formation. However, due to the phenomenon of neglect benevolence (see Section III-F), the optimal input time was not necessarily as early as possible. The argument in [101] is that an aided display is important in such cases because it is difficult 
to perceive the optimal input time by simply looking at the emergent behavior of the swarm. An aided display, informed by the control algorithm, seemed to help operators overcome this issue.

\section{Control Methods-Conveying Operator Intent to the Swarm}

We will now focus on the other side of the control loop: how to properly convey input from the operator to the swarm. Due to the fact that the human control of swarms is desired to be $O(1)$, it stands to reason that in many cases, a swarm can be viewed as a single entity, much as a system with one robot and one human would be, except that the properties and behavior of this system would be very different than that of a single robot. This may not always hold, as some swarms contain heterogeneous members, and some will require splitting into disconnected parts, or giving different members of a swarm different commands. Therefore, there is a need to operationalize the types of control an operator can exert on the swarm. We identify the following types:

1) switching between algorithms that implement desired swarm behaviors;

2) changing parameters of a swarm control algorithm;

3) indirect control of the swarm via environmental influences;

4) control through selected swarm members, typically called leaders.

Within these swarm-specific types of control, we will sometimes distinguish between discrete and continuous inputs. For example, leader-based influence can be achieved with a continuous input to a leader (teleoperation) or with a discrete input. The above types are not mutually exclusive, interact with other properties of the human-swarm system such as the communication scheme (proximal or remote), and they impose varying constraints on the swarm.

1) Algorithm and Behavior Selection: Control via algorithm and behavior selection assumes that the human operator is giving control inputs at discrete time points by selecting a specific swarm algorithm, such as those discussed in Section II-B. It also presupposes that operators have at their disposal a library of algorithms that implement different swarm behaviors. By choosing different algorithms, human control is akin to controlling hybrid systems with the human acting as a switch. During the time that a behavior is active an algorithm, usually a local control law implements the behavior autonomously. A comparison between behavior selection and environmental influence in [102] indicated superior performance for behavior selection for novice operators. Behavior selection was also used in [103] and [104]. Successful control with behavior selection also presupposes that the operator can develop an understanding and has access to an appropriate visualization of the swarm dynamics [101], discussed earlier in Section III-C.

Overall, control via algorithm/behavior selection appears to be an effective method of swarm control when the robots have a high degree of autonomy and can operate largely without error or human oversight in between human inputs. Once instructed to execute a certain behavior, an operator relies on the autonomy of the swarm as well as the autonomy of individual robots to deal with obstacle avoidance, robot-to-robot communication, and local coordination. The transmission of commands from the operator for this type of control does generally not pose significant constraints on the communication network. The greater challenges here relate to the selection of the right behavior, input timing, and state estimation - the operator needs to understand what different swarm behaviors look like in order to employ proper selection and switching.

2) Control via Parameter Setting: Most systems depend on a set of parameters for their operations, and so can many swarm algorithms. The values for these parameters offer a clear avenue for control and influence for an operator, in both discrete and continuous input settings. The key difference for swarms is that parameters do not directly influence the behavior, but rather have indirect effects through behaviors emerging from interactions within the swarm and its environment.

In [24], the wide range of behaviors that can be generated with a simple flocking algorithm given different parameters is presented in great detail. These insights have not yet lead to a human-controlled transition between emergent behaviors by changing the parameters of the system, however. One of the few studies that considered the setting of parameters is found in [105]; yet, it focused on indirect parameter setting aided by an autonomous algorithm rather than allowing an operator to directly modify parameters. Therein, Kira and Potter present preliminary work for a top-down and bottom-up approach for physicomimetic swarm control. For the top-down approach, an operator can set desired global characteristics, such as swarm radius and maximum interagent distance (i.e., a parameter setting interaction). For the bottom-up approach, virtual agents (point particles) are placed in the swarm and interact with it via simulated gravitational forces. Evolutionary computation is then used to learn an appropriate placement and parameterization of these virtual agents to bring about a particular behavior (e.g., a split into two groups). Placement of the virtual particles resembles an environmental interaction (see Section III-D3). The algorithms were tested on a "defend a resource" scenario first in simulation with one resource, six agents, and three virtual particles and also on six Pioneer robotic platforms in the lab. No experiments with human subjects have been reported regarding the effectiveness of this approach.

Another example of parameter setting to control a swarm is found in [106]. Therein, an operator controls a swarm of UAVs, in simulation, by setting the parameters for the "personality" of UAVs, defined by four characteristics: conformity, sociability, dedication, and disposition. These relate to thresholds in a target assignment and bidding process. In addition, the operator can designate regions in the environment as either hot or cold. Hot regions are suggestions to nearby UAVs that this region will contain targets, while cold regions suggest the opposite. Whether a UAV incorporates the operator's suggestion depends on its conformity. There were no user studies carried out in [106], nor any results presented. Some results for a similar system are found [107], but are also lacking user studies.

Despite the examples shown above, parameter setting is most often done during the design state of the swarm, and particular parameters that enable an operator to generate multiple 
emergent behaviors are often desired. An example of this is found in [108]. Therein, the authors investigate the parameter space for a flocking algorithm to determine a set of parameters that allows flocking and torus formations to emerge. An operator then influences a subset of the swarm via teleoperation to switch between flocks and torus formations. The results in [108] indicate that it is easier for an operator to switch from a torus to a flock when the teleoperated robots influence the rest of the swarm via their orientation. These results were obtained using simulation runs in a "Oz of Wizard" style study [109], i.e., with simulated human input.

3) Environmental Influence: One of the distinctly "swarm ish" interaction types is to influence a swarm through environmental factors. Environmental influence involves altering part of the environment, usually virtually, but sometimes physically, to influence the behavior of a swarm within that part. Environmental influence has been implemented as a variety of constructs, including virtual pheromones, virtual beacons, and amorphous computing. The key characteristics of this interaction type are that it is location-dependent and persistent through time (or slowly vanishing in the case of pheromones). Behavior selection in contrast sends a single instruction that can be independent of location and affects robots when it is received and subsequently propagated.

Environmental influence on the swarm is mediated via direct or virtual sensing of environmental changes. Robots in the swarm continue to operate under the same rules they were deployed with and interact with the environment in a consistent manner throughout their operation. It may be argued this is a more suitable way to control the swarm, as it does not directly interfere with the autonomous emergence of different swarm behaviors, i.e., if it can be guaranteed that a behavior will emerge, environmental control should not necessarily affect that guarantee. This, however, depends on the type of environmental influence available, particularly when using virtual pheromones and beacons, and whether the emergent properties are guaranteed in the particular environment.

An example of environmental influence is found in [110]. Therein, the authors use the analogy of a digital display to represent a swarm of robots, whereby each robot represents a "pixel" in the environment and gives information only from its local environment and neighboring robots to a human operator. The example they give is that of a search and rescue scenario inside a building, where a deployed swarm can spread out, and once a victim is identified, the robot viewing them can propagate its information back through the swarm via virtual pheromones to the human operator. In their case, the rescuers can then view the combined information from all nearby robots on a headmounted display as they travel through the environment looking for the victims. Furthermore, the human operator can influence the swarm by injecting pheromone information to nearby robots via a handheld display. Another example of virtual pheromones is given in [111], wherein operators demonstrate the ability to use virtual pheromones to control up to 50000 robots in simulation. Another example of environmental influence is given in [112] and [102], where the authors use simulated beacons that can be placed by an operator and signal to nearby robots to execute a certain behavior. A set of seven different behaviors are implemented. The beacons can be placed anywhere in the environment to allow the operator to modify the overall swarm behavior via the perceived environment as he or she sees fit. Experimental results indicate, however, that behavior selection for the same set of behaviors leads to superior performance, as compared with placing beacons, for untrained operators on a foraging task.

Environmental interactions are also a natural type of interaction mode for amorphous computing algorithms, discussed in more detail in Section II-A3. The advantage that an amorphous computing paradigm provides lies primarily in the enforcement of a local context when writing swarm programs. It also eases the maintenance of a set of variables that requires distributed computation across the swarm medium. In principle, this could enable all types of HSI, but is ideally suited for environmental influence due to its in-built emphasis on spatial distribution. Yet, no studies investigating human control of swarms based on amorphous computing principles have been carried out. The emphasis on expressive instructions that depend on and persist with regard to time and location suggest that there is ample room for investigation.

4) Leader Selection: One method to deal with the complexity of controlling a swarm is to allow an operator to select and control a subset of the swarm, thereby reducing the number of robots that have to be considered simultaneously. Individuals or groups of robots selected by an operator are frequently denoted as leaders since they are expected to influence and lead the remaining swarm, as a proxy for the operator. The selection of a small set of individual robots as leaders opens up the possibility for more engaging forms of control that are also used for single and multi-robot systems, such as teleoperation. The key difference between swarms controlled via leaders and other systems is that leaders have an influence that propagates through the swarm, and an operator should attempt to control the entire swarm via this propagated influence. The main questions for leader-based control are: 1) how to best select the leader; 2) whether a selected leader remains a leader throughout a scenario or whether leadership is transient; 3) how to control for propagation effects on the remaining swarm; and 4) how leaders should interact with nearby swarm members.

Persistent Influence via Leaders: In cases where more precise control over a swarm's operation is needed, or when a desired emergent behavior cannot be generated autonomously and without significant human influence, continuous inputs may be given by a human operator. These continuous inputs will have a persistent influence on selected leaders and indirectly on the swarm, and such situations require significantly more training and attention on the part of the operator. In its basic form, persistent influence is akin to teleoperation. It generally involves some notion of the state of the system fed back to the operator who can then modify the inputs accordingly. Such control usually requires a tight feedback loop with low latency and a representation of the system state that is interpretable for the operator. However, proximal interactions are also conducive to continuous control since the human can always be sensed by the robots continuously and can direct them much like a leader 
robot, and thus, any movement of the operator is potentially an input to the swarm. In Section III-C, we briefly discussed the difficulties of estimating and visualizing the state of a swarm. For controlling motion of single- and multirobot systems, visual and haptic feedback has been used predominantly, and these do not easily translate to swarms. The selection of swarm leaders, however, can enable such control. In this case, the control of a single leader or a group of leaders is similar to single- or multirobot teleoperation. The key difference is the influence of the motion of swarm leader on the remaining swarm that has to be taken into account.

In [113], a leader robot in the swarm is teleoperated in order to aid in the localization of a radiation source. The swarm is influenced indirectly through the motion of the teleoperated robot. The influence is determined by the mode of the robot and can "push" other robots or direct them into one of four directions (up, down, left, right). Once deselected, the robot can be instructed to maintain its mode and thereby its influence on neighboring robots. Results of a small user study indicated that human-operated swarms were significantly better than a fully autonomous swarm at finding the radiation sources within the environment. Goodrich et al. [25], [114]-[116] have also worked extensively on leader-based control of swarms that follow Couzin's control laws [24]. Therein, the authors investigate using teleoperated leaders, which will either attract or repel neighboring robots, to allow a human operator to control the swarm. The authors also consider swarm members, the socalled stakeholders, that are influenced by the operator as well as other swarm members in contrast to the teleoperated leaders (also called predators in the case of repelling leaders). An emphasis is placed on determining under what conditions operator influence can lead to different emergent behaviors and formations. In [117], the authors implement a leader-based model both in simulation and on real robots, using both virtual agents and a human operator as leaders in a swarm, and found that this method scales reasonably well to larger swarm sizes in an information foraging task without obstacles, i.e., it is reasonably close to $O(1)$ type control. In [118], the authors propose two methods for propagating operator intent from a single leader to the rest of the swarm. The first is explicit, where the leader can be distinguished from other neighboring robots, and thus, its neighbors can explicitly follow the leader's heading; and the second is tacit, where the leaders are indistinguishable, and implicitly biases the average speed and heading of neighboring robots. Here, the authors found that the explicit method gave human operators better control over the swarm, but hypothesized that the tacit method could be more robust to sensing error if a larger percentage of the swarm were leader robots, to allow for faster propagation of used intent. In [119] and [120], the authors further this work by presenting an algorithm for selecting multiple leaders dynamically in a swarm as the topology of the communication graph changes. They found that, while the explicit method of propagation was again superior overall, the tacit method performed better under significant sensing error.

The selection of single leaders or small groups of leaders has been the default choice for much of the work on HSI that involves persistent and continuous influence. One of the few exception is found in [121]. Therein, operators used a haptic joystick to give continuous inputs to the entire swarm during a target-searching task. The human teleoperated the swarm via broadcast commands by manipulating the joystick. The swarm itself handled obstacle avoidance and maintenance of proper robot-to-robot distances, but global goal direction and speed of the robots were controlled by the human. The haptic feedback given to the operator is computed as the average of all forces exerted on all swarm robots resulting from repulsion from obstacles, similar to the approach in [48]. The authors found that giving continuous inputs with haptic feedback allowed for superior control and more targets found.

In general, teleoperation of robots has been studied extensively, but the primary emphasis has been on single robots. Here, we are going to review some of the work done for bilateral teleoperation of multirobot systems, for which there is usually a master robot that a human uses to control a slave robotic system. Information is fed back and forth (as forces) between the human and the slave system through the master robot or haptic device. Haptic feedback can be used to augment existing methods like continuous visual feedback. Recent efforts in this area are found in [122]-[124]. These can be broadly put in two categories depending on the communication (and control) architecture between the master and slave systems: 1) centralized approaches-where each robot communicates individually with the master system [122], [123], [125]; and 2) decentralized approaches_-where the robots coordinate among themselves, and only a single robot communicates with the master robot [124], [126]-[128]. Control and communication should ensure safety and stability, i.e., avoid collision and track a desired reference trajectory (e.g., maintaining a certain formation).

A decentralized strategy was proposed by Franchi et al. [124] based on a leader-follower approach where the slaves are assumed to have second-order point mass dynamics [124]. The key contribution of [124] is to design a potential function (and hence a control law) that ensures that the overall system is passive. The controller has been tested with a human controlling a team of up to six simulated UAVs. Although the authors allow the agents to make or break links, there is no guarantee that the connectivity of the robotic network is maintained. In [126] and [129], the authors have extended the work from [124], [127] to ensure that the designed haptic control laws ensure stability even in the presence of delays. Similar techniques have also been used for haptic control of UAV formation where the UAVs only use the bearing information of their neighboring agents [128]. The above control schemes have been limited to either formation control or target tracking. Haptic control schemes for other multirobot tasks (e.g., area search and coverage, foraging, cooperative mapping, etc.) are not available. To apply this work on teleoperation to a swarm, in particular, a selected subgroup of leaders in the swarm, the repercussions and effects of the motion of this subgroup on the overall swarm behavior and dynamics would need to be integrated into the control scheme so that an operator can control the subgroup while being aware of the implications and compensating for the overall swarm behavior.

Discrete Influence via Leaders: Numerous works have implemented discrete control systems in which the operator sends 
messages to selected robots intermittently. This method is easy to implement and requires little training for the human operator. It is also well suited for both homogeneous and heterogeneous swarms, as different commands can be easily and distinctly given to each type of robot. For example, in [104], operators effectively deployed a heterogeneous swarm in an ocean setting to test the viability of swarms in monitoring data in waterways. The operators had sparse intermittent communication with the robots-being able to send and receive data only when the robots surfaced, and sending commands to correct errors in the robots' trajectories due to sensing error and ocean currents.

In [130], the authors present a method for the user to select and assign tasks to a single leader robot out of many in an indoor environment (with a distance between the human and robot between 1 and $4 \mathrm{~m}$ ). Each robot first recognizes how directly the human is looking at it through facial recognition. It then uses a ring-based leader election algorithm to determine the single robot with the highest face detection score. The user then commands this robot with gestures. Pilot experiments with human participants produced encouraging results; yet, it is not clear how the approach scales and how appropriate it is for larger distances between user and robots. Also suitable for discrete control inputs is the work presented in [88], [90], and [91], which enables proximal interactions with operators by transmitting commands to the swarm with gestures, face engagement, and speech.

\section{E. Levels of Autonomation in Human-Swarm Interaction}

In [131], Sheridan and Verplank propose a ten-point level of automation (LOA) scale to characterize the degrees of autonomy possible for human-machine systems, ranging from a system where the machine has full autonomy (10) to one where the human controls everything (1). This scale has been used and modified extensively to describe and evaluate levels of automation for a number of supervisory control systems on differing robotic platforms [132]-[134]. A human-swarm system would fall high on the scale_-greater than or equal to 7. This model for levels of automation has been used in numerous works studying HRI and HSI (see [103] and [135] for recent examples).

One of the earlier discussions on LOAs in swarms is found in [136]. In particular, the author distinguishes between levels of automation within the swarm and levels of automation for the decision making of the operator. Much of the autonomy in a swarm serves the purpose to coordinate the entire system and does not necessarily impact the amount of information an operator has access to nor the level of involvement for certain decisions. Yet, as pointed out in [136], the level of autonomy for coordination does have an indirect impact on the situational awareness of the operator.

In [135], the authors introduce the autonomy spectrum for HSI, which extends the LOA model by allowing different userselectable modes at each control task corresponding to different possible LOAs for each task. Furthermore, their model includes predefined pathways between different LOA combinations at each stage, corresponding to the different possible methods of operation. The approach has been tested with human operators in an elaborate application scenario involving patrolling and pursuit. The primary conclusion is that operators had some positive impact on system performance but that much further work remains to be done to better integrate human and swarm autonomy. Similarly, in [103], the authors use two switchable modes of operation for a swarm to allow the human operator to switch between high and low autonomy. The high autonomy was captured via a dispersion algorithm whereby the swarm members spread to cover the open space in the environment, and the low autonomy mode allowed the user to select subsections of the swarm to direct via waypoints. Here, the authors found that operators found the most success when using a mix of the two modes, instead of solely one or the other.

Further work is needed before we are able to say properly whether flexible levels of automation in HSI are beneficial; however, this preliminary work suggests it could be. Yet, one of the unstudied issues is how humans react to flexible autonomy with swarms. Because the emergent properties of swarms are often unpredictable, and because we have little knowledge of human operators' understanding of swarms, it is possible that introducing task switching between different levels of automation may significantly degrade the situational awareness of operators, and may interfere with their understanding, leading to decreased performance. As this and other cases of LOAs impact nearly all aspects of the control loop, they are essential to investigate if we desire a true understanding of human-swarm systems.

\section{F. Input Timing and Neglect Benevolence}

Not only is the method of giving different commands of concern to human operators and those designing the HSI system, but also the timing of those commands. Since some swarm algorithms require time to converge and stabilize after an operator command is issued, it is possible for the same types of commands to have different-sometimes adverse-effects depending on the state of the swarm. To capture the idea that humans may need to observe the evolution of the swarm state and wait some time before acting, a novel concept called neglect benevolence was investigated. This concept is in some sense the converse to neglect tolerance [137], [138] in HRI of independent and noncoordinating robots, where it is assumed that the performance of an individual robot degrades with time, and hence, the attention of the operator needs to be scheduled so that the time between servicing robots (the neglect time) is minimized [139].

Consider, for example, a generic flocking algorithm. One of the issues that may occur for flocking is the fragmentation of the swarm, and frequent instructions for changes in direction of the flock may lead to such fragmentation, unless the swarm regains its cohesion before the next instruction. The risk of fragmentation is increased by delays in coordination, errors in motion and sensing, as well as external perturbations. In [140], it was shown that improper timing of control input could lead to swarm fragmentation. In [87], the authors show evidence of neglect benevolence in swarms during a simple target-searching task. They found that operators who issued commands frequently showed lower levels of performance than those who allowed the swarm to adjust between new commands. This was the first study to 
give evidence to the concept of neglect benevolence by showing that commands given too frequently to a swarm exhibiting emergent behavior could actually degrade performance.

Neglect benevolence formally defined in [140], where the authors proved the existence of neglect benevolence for linear time-invariant systems, developed an algorithm to determine the optimal input time for such a system. In [101], the authors further investigate human performance in the face of neglect benevolence and showed that human study participants learned to approximate the optimal time over the course of the experiment in a formation control task. Neglect benevolence and optimal timing studies are just beginning to emerge, and they are an interesting area for future research. Additionally, algorithms to determine optimal human timing could be incorporated to provide operator decision support.

\section{DISCUSSION}

In the previous section, we presented and discussed our organizational structure from Fig. 1, centered around the operator's perspective and our set of research questions, and reviewed existing HSI studies within this structure. We also reviewed aspects of a human-swarm system that impact all parts of the control loop (input timing and neglect benevolence and levels of automation). Table I summarizes the key issues for each topic including the context in which it was studied. HSI research is still in its early stages, and identifying the right context and methods for studies is still a challenge, but a few themes have started to emerge. Here, we will briefly discuss these and how they relate to the problem of isolating the various interacting components in complex swarm systems.

Our organization, illustrated in Fig. 1, emphasizes that levels of automation and input timing and neglect benevolence may interact with all components of the human-swarm control loop. As such, they are studied in setups with specific control algorithms, visualizations, swarms, and tasks. Also expressed in our organization is the fact that the communication infrastructure, which supports the interface between human and swarm, has significant influence on state estimation as well as control methods. Hence, research on these two components needs to clarify the underlying assumptions about the communication infrastructure and ideally deal with the implications of limited reliability, connectivity, bandwidth, and latency. State estimation and control methods can be studied somewhat independently, as prior work has demonstrated. In fact, many of the studies to date have been concerned with the interactions between the chain of components from the operator, control methods, up to the swarm. The chain of components from swarm to state estimation and visualization up to the operator has received less attention, particularly how the estimation relates to operator cognition.

Broadly speaking, HSI research has employed three basic methods of inquiry. Theoretical analysis and models have been proven useful to determine feasible and optimal control inputs, e.g., with behavior selection [100] and investigating neglect benevolence [101]. When closed-form solutions are not available, simulations have proven useful, such as in [25]. Finally, user studies and prototype systems in simulation or with real robots are used to address more complex scenarios or verify theoretical insights.

One of the main problems tackled in much of the HSI research is the large and divisible state space of swarms. This problem is also at the core of our cognitive complexity perspective discussed in Section III-A. Consequently, in Section III-D, we have presented control methods that aim to reduce the complexity of controlling a swarm, namely behavior selection, parameter setting, environmental and leader influence. The aim for each of these methods is to enable interactions that scale to large numbers of robots, i.e., $O(1)$ style interactions. All these methods, however, when integrated into complex systems, involve some form of implicit or explicit selection of robots. Leader influence is explicitly concerned with which robots should be the gateways of information between the swarm and operator. Environmental influence selects robots implicitly by determining which robots can sense the real or virtual change in the environment. Behavior selection and parameter setting are swarm-wide controls, but in practice a user often selects a subswarm to which to apply the desired behavior or parameter (with the exception of a few studies). Without selection, these two methods do not allow finegrained control, which on the other hand makes them easier to model, as in [100]. However, even when using leader influence, current methods do not allow the swarm to solve complex tasks, and these will likely also involve a division into subswarms.

In fact, the formation of subgroups is a common feature in studies with complex application scenarios as in [102], [103], and [135], and envisioned in [88], [90], and [91]. Therefore, it can be argued that the problem of dividing a swarm into subswarms is superimposed over our control taxonomy and that most operators are going to be confronted with the issue of selecting and managing multiple groups of swarm robots. Once operator-controlled selection of robots is permitted, in the best case, $n$ swarm robots become $k$ independently operating swarms and their management would be $O(k)$. In this case, the multirobot concept of neglect tolerance and fan-out models would apply. In the worst case, robots in different subswarms continue to interact and interfere with each other and management becomes $O(>k)$. Now, $k$ may not depend on $n$, but instead on the task at hand, e.g., when viewing the swarm as an approximation of a continuous medium while using environmental influence.

At this point, it becomes important how a control method performs when it controls a subswarm that is embedded in a larger swarm. In particular, one should determine the consequences of interactions between multiple subswarms. This sort of investigation is very much needed for future work. Each individual interaction should be still be an $O(1)$ style interaction within an overall system that may become more complex. Our presented framework also provides the means to study the individual methods in isolation and generalize to the broader context. For example, one can investigate the neglect benevolence and neglect tolerance properties of a particular interaction method, such as a particular behavior selection scheme, and then study the consequence of embedding said scheme into a larger system that allows multiple subswarms. At this point, methods from human interaction with multirobot systems could be applied to, e.g., schedule the operator's attention to individual 
TABLE I

SUMmaRY TABLE FOR OUR SECTION ORGANIZATION, INCLUDING THE CONTEXTS IN WHICH HSI TOPICS ARE STUDIED

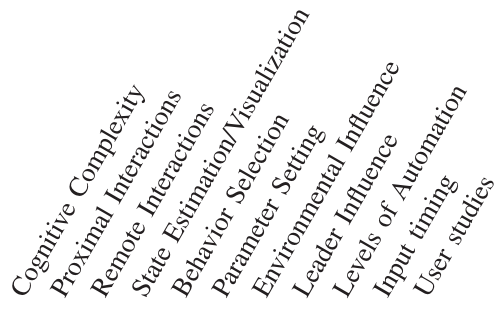

Cognitive complexity

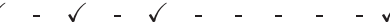

i.e.: studied with remote interactions \& behavior selection through user studies

A general framework [70], [3] for scalable human control to deal with large state spaces and complex dynamics; basic swarm control schemes aim to be $O(1)$; extensions to other cognitive factors have been considered in the context of remote interactions and behavior selection with formal models of cognition [99], [100];

Proximal Interactions

Largely studied with real robots and focused on enabling any interaction at all; current results enable relaying of instructions [130], [88], [89], [90], [91]; a key challenge is to scale these to multiple operators, large environments and swarms, as well as to conduct comprehensive user studies;

Remote Interactions

- $\quad \checkmark \checkmark \checkmark-\quad-\quad-\quad-\checkmark$

Most user studies assume remote interactions; latency [87] and bandwidth [86] limitations have been investigated in the context of behavior selection; connectivity maintenance and loss was possible in [102]; communication constraints can require the use of aggregate statistic that may be sufficient for effective control [96]; adaptive networks still have to be investigated; the key challenge to reconcile a distributed remote network with a central operator underlies many HSI issues.

State Estimation/Visualization $-\checkmark \checkmark \checkmark \checkmark-\quad-\checkmark \checkmark \checkmark \checkmark$

Key issues are predicting consequences of control actions, understanding of swarm dynamics as well as aggregate statistics; studied in a number of contexts such as communication constraints, leader influence, and behavior selection [96], [87], [86]; predictive displays have been proven useful for behavior selection [87], [101]; aggregate state descriptions can be sufficient in some scenarios; no work on general human perception of swarms, e.g., involving Gestalt principles, is available.

Behavior Selection

The simplest form of swarm control, behavior selection has been considered in a number of contexts [113], [112], [102], [103]; a comparison between behavior selection and environmental influence revealed superior performance and significant differences in the strategies employed by operators [102]; a crucial consideration is the timing of instructions, time to convergence of emergent behaviors, and whether subgroups of robots are permitted to run different algorithms leading to heterogeneous swarms, causing a possible increase in cognitive complexity.

Parameter Setting

$-\quad-\quad-\quad \checkmark-\quad-\quad-\quad$

A form of swarm control not investigated in detail in user studies, but typically used during the design stage [25] or with automation [105], [106], [107];

Environmental Control

$-\quad-\checkmark \checkmark-\checkmark-\quad-\checkmark$

Swarm control via real or virtual changes in the environment sensed by the swarm; first studies in [102] revealed this form of control to be more difficult for novice operators than behavior selection; [135] also reports difficulties interacting with pheromone controlled swarms; also used in [110], [111] with visualizations of pheromones but generally less studied than behavior selection and leader influence; potentially a suitable control method for the problem of controlling many sub-swarms;

Leader Influence

Control complexity is reduced by controlling leaders that influence the swarm; key questions are the selection of leaders and propagation of influence; a comparison between strong or weak influence is found in [118]; algorithmic dynamic selection of leaders improves control over the swarm [120], [119]; swarms with parameters optimized for allowing human controlled transitions between emergent behaviors are used in [25], [117], [108]; Teleoperation of selected leader groups in simulated and real swarms is feasible and enabled by [123], [124], [125], [126], [127], [128].

Levels of automony

$-\quad-\checkmark-\checkmark \checkmark-\checkmark \checkmark-\checkmark$

First mentioned for swarms in [136]; introduction of autonomy spectrum in [135] which connects levels of automation to task sequences; results are focused on performance in a specific application scenario with the conclusion that more HSI research is warranted to close the representation gap between swarm and operator intelligence; [103] suggests that levels of automation should be dependent on environmental conditions; despite the high reliance of swarms on autonomy the impact of varying levels of automation has not been studied extensively;

Neglect benevolence

$-\quad-\checkmark-\checkmark-\checkmark-\checkmark \checkmark$

Neglect benevolence is a swarm-specific concept that is concerned with input timing and the resulting impact on the swarm state; Potentially disturbances of stable states and emergent behaviors by poorly timed human inputs are at the core; Neglect benevolence was first observed in [87] and analyzed in more detail in [140]; user studies showed that operators are able to approximate optimal input timing [101]; neglect benevolence is a useful concept for swarms to reinforce the importance of temporal dynamics for HSI designers. 
subswarms appropriately. The study of individual interaction schemes for behavior selection and leader influence has potentially progressed to a stage where such investigations and experiments are made possible. Moreover, we conjecture that leader influence methods will likely lead to fewer interferences between subswarms but will also be less powerful within a larger system and suffer more from neglect tolerance. Behavior selection on the other hand will lead to larger interference between subswarms, may suffer due to high neglect benevolence, but will likely scale better to many subswarms and benefit from better neglect tolerance.

The management of multiple subswarms also offers an insight into the qualitative differences within our control taxonomy. Behavior selection influences all members of a subswarm, leader influence only a few, and environmental influence implicitly creates and influences new subswarms. Hence, environmental influence interacts differently with the problem of subswarms and has the potential to deal directly with subswarm hierarchies due to its implicit selection mechanism. Because there are few studies using this control approach, we believe more are needed before we can really make determinations about the interaction between multiple subswarms using environmental influence.

\section{FUTURE WORK AND CONCLUSION}

This review is meant for researchers that are attempting to further the field of HSI. Hence, we gave a brief introduction to swarm robotics to provide an overview of the kinds of properties one should expect of swarm systems, as well as an introduction to HSI structured and centered around the operator. We began with a discussion on cognitive complexity, and then with an examination of the control loop, with particular attention to communication, perceiving the swarm, and exerting control. Finally, we discussed some overarching issues that pertain to the entire control loop in sections on levels of automation as well as input timing and neglect benevolence. Throughout, we have noted a number of challenges, in our discussion as well as within each specific category, some of which have been addressed, but most of which remain unsolved. Here, we briefly summarize what we envision as the main challenges, in addition to the management of subswarms discussed in the previous section, that HSI research can address in the near future based on the current state of HSI research. These are related to the three main sources of difficulty in swarm control: tasks, communication, and uncertainty and size of the state space. Some of the resulting challenges can be addressed fairly independently of each other and were also discussed in the previous sections.

\section{A. Suitability of Control Type Relative to Task and Environment}

A set of important research questions for HSI relates to the characteristics of the control types. It is essential to determine which general types and their various implementations are suitable for what kinds of tasks, environments, communication and timing constraints, and other swarm-specific circumstances. In addition, when multiple types are suitable, one should attempt to compare effectiveness, scope, and impact of these control types, i.e., how many robots they affect directly and indirectly.
In addition, entirely novel interaction techniques, beyond those we presented in this paper, may also have to be investigated when the existing types fall short.

What we know so far about the characteristics of the control types is fairly limited. Behavior selection is perhaps a natural choice for many swarm applications, suitable for untrained operators and shown to be superior to environmental control, particularly when existing behaviors already solve the tasks. Yet, it is likely to be more affected by neglect benevolence, depending on the behaviors available. As a consequence, it benefits strongly from predictive displays that aid the operator in determining the effects of commands. On the other hand, it may well be more robust to communication issues, i.e., latency, bandwidth, and asynchrony.

Environmental interactions, however, appear to be a particularly swarm-like type of interaction. This becomes more apparent when considering a swarm as an approximation of an amorphous medium. The complexity of controlling it does not scale with the number of robots but rather the size of the medium and environment. Regarding this type of interaction, little work has been done so far; yet, it seems a fruitful area for further HSI research. It may well be that effective environmental interactions require operator training and more advanced interfaces than the simpler interaction types.

Another difficulty in swarm control that has not received much attention is how to enable effective leader-based control. Predicting, limiting, or correcting for leader propagated influence is one immediate issue. In addition, direct control of leaders requires a low latency network. As such, it is a more difficult type of control to implement reliably for swarms and no studies have been carried out with real swarms.

\section{B. Swarm Visualization and Understanding of Dynamics}

Humans are structured to find groups and patterns in everyday life, and these skills could potentially be applied to swarms as well. Because swarms often operate under emergent behaviors and large-scale group patterns that come from local interactions, humans may be uniquely suited to identify, categorize, and alter the global swarm behavior. It is an open research area whether it is possible to design algorithms having in mind human undestandability of their results. Some work, discussed in Section III-C, has been carried out to investigate visualization of swarm states and behavior, but little is known with regard to general principles of swarm visualization and perception by operators, particularly for noisy or partially accessible states. This is especially important for an operator's understanding of swarm dynamics that unfold over time and therefore provide a further challenge for visualization. Exactly what information an operator needs access to and how it should be visualized for the various swarm algorithms and tasks is, therefore, an important question for swarms, particularly if 1) it can reduce the amount of state information that the swarm needs to communicate to the operator and 2) aid in human understandability of swarm dynamics.

\section{Input timing and Neglect Benevolence}

We have discussed the issue of input timing and the resulting concept of neglect benevolence in some detail in Section III-F. A 
few studies have begun to emerge that approached the issue from a formal perspective as well as from an experimental perspective, albeit in simulation. Neglect benevolence and optimal input timing seem to be particularly relevant for swarms and may well be exhibited by many of the algorithms currently envisioned for use in real swarm applications. In particular, for control with behavior selection, the dominating issue becomes input timing and the prediction of swarm dynamics. Further studies are required to determine under which conditions, human operators can learn to time their inputs optimally.

\section{Cognitive Complexity and Levels of Automation}

In the existing human interaction literature, cognitive complexity was primarily considered in terms of cognitive effort in relation to the number of robots. Task difficulty was primarily dealt with when considering workload, fatigue, and other human factors issues, while levels of automation dealt with access to information and decision-making roles. Rarely are these three issues considered jointly, and swarms present a suitable context in which to investigate the interactions between these as well as novel notions of cognitive complexity that take into account system size, task difficulty, levels of automation, and their respective interactions. One early study that points in the right direction is conducted in [135], subsequently extended in [103], and has been discussed in prior sections. This is clearly an area that warrants further work to move toward real swarms solving more complex missions and tasks.

\section{E. Swarm Metrics and Experiments}

Most of the guarantees and metrics that have been developed thus far are concerned with convergences to an emergent goal behavior for a particular task under specific assumptions. Reliable metrics regarding the performance of a swarm in real applications are not available, apart from some early results in [141] and [142], and their general need is identified in several swarm surveys [5]-[7]. From the perspective of HSI, this is a promising area of research, and when monitoring swarms, operators can greatly benefit from such metrics, e.g., when managing competing tasks while monitoring the quality of service in the communication network. These metrics can also help by overcoming the problem with running HSI studies in practice, particularly the lack of studies with real robot systems. Measuring and monitoring the behavior of real swarms will also enable the reproduction of more realistic swarm behavior in simulation. On the other hand, it is also desirable to have more studies with real robots and real human operators to provide a firm foundation for HSI studies.

In this paper, we have attempted to draw the outlines of HSI research, a young subdiscipline and one not yet well understood. Experimental studies in cooperation with researchers in swarm robotics and the anticipated development of swarms outside the confines of laboratories will inevitably shed further light onto the topic.

\section{REFERENCES}

[1] A. Farinelli, L. Iocchi, and D. Nardi, "Multirobot systems: a classification focused on coordination," IEEE Trans. Syst. Man, Cybern. B, Cybern., vol. 34, no. 5, pp. 2015-2028, Oct. 2004.
[2] L. E. Parker, "Multiple mobile robot systems," in Springer Handbook of Robotics. New York, NY, USA: 2008, pp. 921-941.

[3] M. Lewis, "Human interaction with multiple remote robots," Rev. Human Factors Ergonomics, vol. 9, no. 1, pp. 131-174, 2013.

[4] E. Bonabeau, M. Dorigo, and G. Theraulaz, Swarm Intelligence: From Natural to Artificial Systems, vol. 4. New York, NY, USA: Oxford Univ. Press, 1999.

[5] J. C. Barca and Y. A. Sekercioglu, "Swarm robotics reviewed," Robotica, vol. 31, no. 03, pp. 345-359, 2013.

[6] M. Brambilla, E. Ferrante, M. Birattari, and M. Dorigo, "Swarm robotics: a review from the swarm engineering perspective," Swarm Intell., vol. 7, no. 1, pp. 1-41, 2013.

[7] Y. Tan and Z.-Y. Zheng, "Research advance in swarm robotics," Defence Technol., vol. 9, no. 1, pp. 18-39, 2013.

[8] W. Truszkowski, M. Hinchey, J. Rash, and C. Rouff, "NASA's swarm missions: The challenge of building autonomous software," IT Professional, vol. 6, no. 5, pp. 47-52, 2004.

[9] R. Vaughan, "Massively multi-robot simulation in stage," Swarm Intel., vol. 2, no. 2, pp. 189-208, 2008

[10] Micro Autonomous Systems and Technology_Collaborative Technology Alliance. (2011). [Online]. Available: http://www.mast-cta.org

[11] Swarmanoid: Towards Humanoid Robotic Swarms-EU IST-FET Project. (2011). [Online]. Available: http://www.swarmanoid.org

[12] F. Mondada, G. C. Pettinaro, A. Guignard, I. W. Kwee, D. Floreano, J.-L. Deneubourg, S. Nolfi, L. M. Gambardella, and M. Dorigo, "SwarmBot: A new distributed robotic concept," Auton. Robot., vol. 17, no. 2-3, pp. 193-221, 2004.

[13] M. Dorigo, V. Trianni, E. Sahin, R. Groß, T. H. Labella, G. Baldassarre, S. Nolfi, J.-L. Deneubourg, F. Mondada, D. Floreano, and L. M. Gambardella, "Evolving self-organizing behaviors for a swarm-Bot," Auton. Robot., vol. 17, nos. 2/3, pp. 223-245, 2004

[14] R. O'Grady, R. Groß, A. L. Christensen, and M. Dorigo, "Self-assembly strategies in a group of autonomous mobile robots," Auton. Robots, vol. 28 , no. 4, pp. 439-455, 2010.

[15] H. Çelikkanat and E. Şahin, "Steering self-organized robot flocks through externally guided individuals," Neural Comput. Appl., vol. 19, no. 6 pp. 849-865, 2010.

[16] M. Rubenstein, C. Ahler, N. Hoff, A. Cabrera, and R. Nagpal, "Kilobot: A low cost robot with scalable operations designed for collective behaviors," Robot. Auton. Syst., vol. 62, no. 7, pp. 966-975, 2014.

[17] M. Rubenstein, A. Cornejo, and R. Nagpal, "Programmable selfassembly in a thousand-robot swarm," Science, vol. 345 , no. 6198 pp. 795-799, 2014.

[18] G. Dudek, M. Jenkin, and E. Milios, "A taxonomy of multirobot systems," in Robot Teams, T. Balch and L. Parker, Eds., Wellesley, MA USA: AK Peters, 2002, pp. 3-22.

[19] Y. U. Cao, A. S. Fukunaga, and A. B. Kahng, "Cooperative mobile robotics: Antecedents and directions," Auton. Robot., vol. 4, no. 1, pp. 7-23, Mar. 1997.

[20] L. Parker, "Current research in multirobot systems," Artif. Life Robot., vol. 7, no. 1, pp. 1-5, 2003.

[21] Y. Mohan and S. Ponnambalam, "An extensive review of research in swarm robotics," in Proc. World Congr. Nature Biol. Inspired Comput., 2009, pp. 140-145.

[22] P. Holmes, R. Full, D. Koditschek, and J. Guckenheimer, "The dynamics of legged locomotion: Models, analyses, and challenges," Siam Rev., vol. 48, pp. 207-304, 2006.

[23] R. Arkin, Behavior-Based Robotics. Cambridge, MA, USA: MIT Press, 1998.

[24] I. D. Couzin, J. Krause, R. James, G. D. Ruxton, and N. R. Franks, "Collective memory and spatial sorting in animal groups," J. Theor. Biol., vol. 218, no. 1, pp. 1-11, 2002.

[25] M. A. Goodrich, B. Pendleton, S. Kerman, and P. Sujit, "What types of interactions do bio-inspired robot swarms and flocks afford a human?" in Proc. Robot.: Sci. Syst. VIII, 2013, pp. 105-112.

[26] J. Sauter, S. Robert, A. Mathews, and S. Riddle, "Distributed pheromonebased swarming control of unmanned air and ground vehicles for RSTA," Ann. Arbor., vol. 1001, p. 48105, 2008.

[27] J. A. Sauter, R. S. Matthews, J. S. Robinson, J. Moody, and S. P. Riddle, "Swarming unmanned air and ground systems for surveillance and base protection," presented at the AIAA Infotech@ Aerospace Conf., Seattle, WA, USA, 2009.

[28] D. Payton, M. Daily, R. Estowski, M. Howard, and C. Lee, "Pheromone robotics," Auton. Robot., vol. 11, no. 3, pp. 319-324, 2001.

[29] D. Sumpter, "The principles of collective animal behaviour," Philosophical Trans. Royal Soc. B: Biol. Sci., vol. 361, no. 1465, pp. 5-22, 2006. 
[30] M. Steinberg, "Biologically-inspired approaches for self-organization, adaptation, and collaboration of heterogeneous autonomous systems," Proc. SPIE, vol. 8062, p. 80620H, 2011.

[31] V. Gazi and B. Fidan, "Coordination and control of multi-agent dynamic systems: Models and approaches," in Swarm Robotics (ser. Lecture Notes in Computer Science), vol. 4433, E. Sahin, W. Spears, and A. Winfield, Eds. Berlin, Germany: Springer, 2007, pp. 71-102.

[32] F. Bullo, J. Cortés, and S. Martinez, Distributed Control of Robotic Networks: A Mathematical Approach to Motion Coordination Algorithms. Princeton, NJ, USA: Princeton Univ. Press, 2009.

[33] G. Notarstefano, K. Savla, F. Bullo, and A. Jadbabaie, "Maintaining limited-range connectivity among second-order agents," in Proc. Amer. Control Conf., 2006, pp. 6-pp.

[34] J. Cortés, S. Martínez, and F. Bullo, "Robust rendezvous for mobile autonomous agents via proximity graphs in arbitrary dimensions," IEEE Trans. Autom. Control., vol. 51, no. 8, pp. 1289-1298, Aug. 2006.

[35] J. Cortes, S. Martinez, T. Karatas, and F. Bullo, "Coverage control for mobile sensing networks," in Proc. IEEE Int. Conf. Roboti. Autom., vol. 2, 2002, pp. 1327-1332.

[36] A. Ganguli, J. Cortés, and F. Bullo, "Visibility-based multi-agent deployment in orthogonal environments," in Proc. Amer. Control Conf., 2007, pp. 3426-3431.

[37] S. Susca, F. Bullo, and S. Martínez, "Monitoring environmental boundaries with a robotic sensor network," IEEE Trans. Control Syst. Technol., vol. 16, no. 2, pp. 288-296, Mar. 2008.

[38] S. Martínez and F. Bullo, "Optimal sensor placement and motion coordination for target tracking," Automatica, vol. 42, no. 4, pp. 661-668, 2006.

[39] J.-P. de la Croix and M. Egerstedt, "A control Lyapunov function approach to human-swarm interactions," in Proc. Amer. Control Conf., 2015, pp. 4368-4373.

[40] H. Abelson, D. Allen, D. Coore, C. Hanson, G. Homsy, T. Knight Jr, R. Nagpal, E. Rauch, G. Sussman, and R. Weiss, "Amorphous computing," Commun. ACM, vol. 43, no. 5, pp. 74-82, 2000.

[41] H. Abelson, J. Beal, and G. J. Sussman, “Amorphous computing," MIT Computer Science and Artificial Intelligence Laboratory, Cambridge, MA, USA, Tech. Rep. MIT-CSAIL-TR-2007-030, 2007.

[42] J. Bachrach and J. Beal, "Programming a sensor network as an amorphous medium," MIT Computer Science and Artificial Intelligence Laboratory, Tech. Rep. MIT-CSAIL-TR-2006-069, 2006.

[43] J. Bachrach, J. McLurkin, and A. Grue, "Protoswarm: A language for programming multi-robot systems using the amorphous medium abstraction," in Proc. 7th Int. Joint Conf. Auton. Agents Multiagent Syst., 2008, pp. 1175-1178.

[44] J. Bachrach, J. Beal, and J. McLurkin, "Composable continuous-space programs for robotic swarms," Neural Comput. Appl., vol. 19, no. 6, pp. 825-847, 2010.

[45] T. Vicsek, A. Czirók, E. Ben-Jacob, I. Cohen, and O. Shochet, "Novel type of phase transition in a system of self-driven particles," Phys. Rev. Lett., vol. 75, no. 6, pp. 1226-1229, 1995.

[46] A. Jadbabaie, J. Lin, and A. Morse, "Coordination of groups of mobile autonomous agents using nearest neighbor rules," IEEE Trans. Autom. Control., vol. 48, no. 6, pp. 988-1001, Jun. 2003.

[47] J. Reif and H. Wang, "Social potential fields: A distributed behavioral control for autonomous robots," Robot. Auton. Syst., vol. 27, no. 3, pp. 171-194, 1999.

[48] A. Howard, M. Mataric, and G. Sukhatme, "Mobile sensor network deployment using potential fields: A distributed, scalable solution to the area coverage problem," in Distributed Autonomous Robotic Systems, vol. 5, H. Asama, T. Arai, T. Fukuda, and T. Hasegawa, Eds., Fukuoka, Japan: Springer, 2002, pp. 299-308.

[49] W. Spears, D. Spears, R. Heil, W. Kerr, and S. Hettiarachchi, "An overview of physicomimetics," in Swarm Robotics (ser. Lecture Notes in Computer Science), vol. 3342, E. Sahin and W. Spears, Eds. Berlin, Germany: Springer, 2005, pp. 84-97.

[50] R. Wiegand, M. Potter, D. Sofge, and W. Spears, "A generalized graph-based method for engineering swarm solutions to multiagent problems," in Proc. Parallel Problem Solving Nature-PPSN IX, 2006, pp. 741-750.

[51] W. M. Spears and D. F. Spears, Physicomimetics: Physics-Based Swarm Intelligence. New York, NY, USA: Springer, 2012.

[52] W. M. Spears, D. F. Spears, J. C. Hamann, and R. Heil, "Distributed, physics-based control of swarms of vehicles," Auton. Robot., vol. 17, nos. 2/3, pp. 137-162, 2004

[53] W. M. Spears, R. Heil, D. F. Spears, and D. Zarzhitsky, "Physicomimetics for mobile robot formations," in Proc. 3rd Int. Joint Conf. Auton. Agents Multiagent Syst.-, vol 3, 2004, pp. 1528-1529.
[54] P. F. Damasceno, M. Engel, and S. C. Glotzer, "Predictive self-assembly of polyhedra into complex structures," Science, vol. 337, no. 6093, pp. 453-457, 2012.

[55] K. L. Young, M. L. Personick, M. Engel, P. F. Damasceno, S. N. Barnaby, R. Bleher, T. Li, S. C. Glotzer, B. Lee, and C. A. Mirkin, "A directional entropic force approach to assemble anisotropic nanoparticles into superlattices," Angewandte Chemie International Edition, vol. 52, no. 52, pp. 13980-13984, 2013.

[56] S. Camazine, Self-Organization in Biological Systems. Princeton., NJ, USA: Princeton Univ. Press, 2003.

[57] V. Trianni, R. Groß, T. H. Labella, E. Şahin, and M. Dorigo, "Evolving aggregation behaviors in a swarm of robots," in Advances in Artificial Life. New York, NY, USA: Springer, 2003, pp. 865-874.

[58] J. Yu, S. M. LaValle, and D. Liberzon, "Rendezvous without coordinates," IEEE Trans. Autom. Control, vol. 57, no. 2, pp. 421-434, Feb. 2012.

[59] J. McLurkin and J. Smith, "Distributed algorithms for dispersion in indoor environments using a swarm of autonomous mobile robots," in Distributed Autonomous Robotic Systems, vol. 6. Fukuoka, Japan: Springer, 2007, pp. 399-408.

[60] C. W. Reynolds, "Flocks, herds and schools: A distributed behavioral model," ACM SIGGRAPH Comput. Graph., vol. 21, no. 4, pp. 25-34, 1987.

[61] R. Vaughan, N. Sumpter, J. Henderson, A. Frost, and S. Cameron, "Experiments in automatic flock control," Robot. Auton. Syst., vol. 31, no. 1, pp. 109-117, 2000.

[62] E. Ferrante, A. E. Turgut, C. Huepe, A. Stranieri, C. Pinciroli, and M. Dorigo, "Self-organized flocking with a mobile robot swarm: A novel motion control method," Adaptive Behavior, vol. 20, no. 6, pp. 460-477, 2012.

[63] R. Olfati-Saber, "Flocking for multi-agent dynamic systems: Algorithms and theory," IEEE Trans. Autom. Control, vol. 51, no. 3, pp. 401-420, Mar. 2006.

[64] H. Tanner, A. Jadbabaie, and G. Pappas, "Stable flocking of mobile agents, part i: Fixed topology," in Proc. 42nd IEEE Conf. Decision Control, 2003, vol. 2, pp. 2010-2015.

[65] H. Tanner, A. Jadbabaie, and G. Pappas, "Stable flocking of mobile agents, part ii: Dynamic topology," in Proc. 42nd IEEE Conf. Decision Control, 2003, vol. 2, pp. 2016-2021.

[66] J.-L. Deneubourg, S. Aron, S. Goss, and J. M. Pasteels, "The selforganizing exploratory pattern of the argentine ant," J. Insect Behavior, vol. 3, no. 2, pp. 159-168, 1990.

[67] J. Chen, M. Gauci, W. Li, A. Kolling, and R. Gros, "Occlusion-based cooperative transport with a swarm of miniature mobile robots," IEEE Trans. Robot., vol. 31, no. 2, pp. 307-321, Apr. 2015.

[68] A. F. Winfield, "Foraging robots," in Encyclopedia of Complexity and Systems Science, R. A. Meyers, Ed. New York, NY, USA: Springer, 2009, pp. 3682-3700.

[69] F. Ducatelle, G. A. Di Caro, C. Pinciroli, and L. M. Gambardella, "Selforganized cooperation between robotic swarms," Swarm Intell., vol. 5, no. 2, pp. 73-96, 2011.

[70] M. Lewis, J. Wang, and P. Scerri, "Teamwork coordination for realistically complex multi robot systems," in Proc. NATO Symp. Human Factors Uninhabited Military Vehicles Force Multipliers, 2006, pp. 112.

[71] D. R. Olsen Jr and S. B. Wood, "Fan-out: measuring human control of multiple robots," in Proc. SIGCHI Conf. Human Factors Comput. Syst., 2004, pp. 231-238.

[72] Y. Xu, T. Dai, K. Sycara, and M. Lewis, "Service level differentiation in multi-robots control," in Proc.IEEE/RSJ Int. Conf. Intell. Robot. Syst., 2010, pp. 2224-2230.

[73] Y. Xu, T. Dai, K. P. Sycara, and M. Lewis, “A mechanism design model in robot-service-queue control with strategic operators and asymmetric information," in Proc. 51st IEEE Conf. Decision Control, 2012, pp. 6113-6119.

[74] S.-Y. Chien, H. Wang, M. Lewis, S. Mehrotra, and K. Sycara, "Effects of alarms on control of robot teams," in Proc. Human Factors Ergonomics Soc. Ann. Meeting, 2011, pp. 434-438.

[75] S.-Y. Chien, M. Lewis, S. Mehrotra, N. Brooks, and K. Sycara, "Scheduling operator attention for multi-robot control," in Proc. IEEE/RSJ Int. Conf. Intell. Robot. Syst., 2012, pp. 473-479.

[76] S.-Y. Chien, M. Lewis, S. Mehrotra, and K. Sycara, "Effects of unreliable automation in scheduling operator attention for multi-robot control," in Proc. IEEE Int. Conf. Syst. Man Cybern., 2012, pp. 321-326.

[77] J. Wang and M. Lewis, "Assessing coordination overhead in control of robot teams," in Proc. IEEE Int. Conf. Syst. Man Cybern., 2007, pp. 2645-2649. 
[78] S. T. Hayes and J. A. Adams, "Human-swarm interaction: Sources of uncertainty," in Proc. 2014 ACM/IEEE Int. Conf. Human-Robot Interaction, 2014, pp. 170-171.

[79] J. Yick, B. Mukherjee, and D. Ghosal, "Wireless sensor network survey," Comput. Netw., vol. 52, no. 12, pp. 2292-2330, 2008.

[80] I. F. Akyildiz, W. Su, Y. Sankarasubramaniam, and E. Cayirci, "Wireless sensor networks: A survey," Comput. Netw., vol. 38, no. 4, pp. 393-422, 2002.

[81] S. Basagni, M. Conti, S. Giordano, and I. Stojmenovic, Mobile Ad Hoc Networking. New York, NY, USA: Wiley, 2004.

[82] C. E. Perkins, Ad Hoc Networking. Reading, MA, USA: Addison-Wesley, 2008.

[83] R. S. Peterson and E. G. Sirer, "Antfarm: Efficient content distribution with managed swarms," in Proc. 6th USENIX Symp. Netw. Syst. Design Implementation, 2009, pp. 107-122.

[84] M. Saleem, G. A. Di Caro, and M. Farooq, "Swarm intelligence based routing protocol for wireless sensor networks: Survey and future directions," Inf. Sci., vol. 181, no. 20, pp. 4597-4624, 2011.

[85] J. McLurkin, J. Smith, J. Frankel, D. Sotkowitz, D. Blau, and B. Schmidt, "Speaking swarmish: Human-robot interface design for large swarms of autonomous mobile robots," in Proc. AAAI Spring Symp., 2006, pp. 7275.

[86] S. Nunnally, P. Walker, A. Kolling, N. Chakraborty, M. Lewis, K. Sycara, and M. Goodrich, "Human influence of robotic swarms with bandwidth and localization issues," in Proc. IEEE Int. Conf. Syst. Man Cybern., 2012, pp. 333-338.

[87] P. Walker, S. Nunnally, M. Lewis, A. Kolling, N. Chakraborty, and K. Sycara, "Neglect benevolence in human control of swarms in the presence of latency," in Proc. IEEE Int. Conf. Syst., Man Cybern., 2012, pp. 3009-3014.

[88] A. Giusti, J. Nagi, L. M. Gambardella, S. Bonardi, and G. A. Di Caro, "Human-swarm interaction through distributed cooperative gesture recognition," in Proc. 17th Ann. ACM/IEEE Int. Conf. Human-Robot Interaction, 2012, pp. 401-402.

[89] A. Giusti, J. Nagi, L. M. Gambardella, and G. A. Di Caro, "Distributed consensus for interaction between humans and mobile robot swarms," in Proc. 11th Int. Conf. Autonomous Agents Multiagent Syst.-, 2012, vol. 3, pp. 1503-1504.

[90] J. Nagi, A. Giusti, L. M. Gambardella, and G. A. Di Caro, "Humanswarm interaction using spatial gestures," in Proc. IEEE/RSJ Int. Conf. Intell. Robot. Syst., 2014, pp. 3834-3841.

[91] S. Pourmehr, V. M. Monajjemi, R. Vaughan, and G. Mori, ““'you two! take off!": Creating, modifying and commanding groups of robots using face engagement and indirect speech in voice commands," in Proc. IEEE/RSJ Int. Conf. Intell. Robot. Syst., 2013, pp. 137-142.

[92] A. Naghsh, J. Gancet, A. Tanoto, and C. Roast, "Analysis and design of human-robot swarm interaction in firefighting," in Proc. 17th IEEE Int. Symp. Robot Human Interactive Commun., 2008, pp. 255260.

[93] L. Alboul, J. Saez-Pons, and J. Penders, "Mixed human-robot team navigation in the guardians project," in Proc. IEEE Int. Workshop Safety, Security Rescue Robot., 2008, pp. 95-101.

[94] M. A. Goodrich and A. C. Schultz, "Human-robot interaction: A survey," Foundations Trends Human-Comput. Interaction, vol. 1, no. 3, pp. 203-275, 2007.

[95] M. M. Loper, N. P. Koenig, S. H. Chernova, C. V. Jones, and O. C. Jenkins, "Mobile human-robot teaming with environmental tolerance," in Proc. 4th ACM/IEEE Int. Conf. Human Robot Interaction, 2009, pp. 157-164.

[96] D. S. Brown, S. C. Kerman, and M. A. Goodrich, "Limited bandwidth recognition of collective hehaviors in bio-inspired swarms," in Proc. 13th Int. Conf. Auton. Agents Multiagent Syst., 2014, pp. 405-412.

[97] S. Kerman, D. Brown, and M. Goodrich, "Supporting human interaction with robust robot swarms," in Proc. 5th Int. Symp. Resilient Control Syst., 2012, pp. 197-202.

[98] E. Haas, M. Fields, S. Hill, and C. Stachowiak, "Extreme scalability: Designing interfaces and algorithms for soldier-robotic swarm interaction," DTIC Document, Tech. Rep., 2009.

[99] B. Tabibian, M. Lewis, C. Lebiere, N. Chakraborty, K. Sycara, S. Bennati, and M. Oishi, "Towards a cognitively-based analytic model of human control of swarms," in Proc. AAAI Spring Symp. Series, 2014. [Online]. Available: http://www.aaai.org/ocs/index.php/ SSS/SSS14/paper/view/7764.

[100] K. Sycara, C. Lebiere, Y. Pei, D. Morrison, Y. Tang, and M. Lewis, "Abstraction of analytical models from cognitive models of human control of robotic swarms," presented at the 13th Int. Conf. Cognitive Model. Groningen, Germany, 2015.

[101] S. Nagavalli, N. Chakraborty, and K. Sycara, "A study on the bounds of neglect benevolence in input timing for human interaction with robotic swarms," in Proc. ACM/IEEE Int. Conf. Human-Robot Interaction, 2015, pp. 197-204.

[102] A. Kolling, K. Sycara, S. Nunnally, and M. Lewis, "Human swarm interaction: An experimental study of two types of interaction with foraging swarms," J. Human-Robot Int., vol. 2, no. 2, pp. 103-128, 2013.

[103] P. Walker, S. Nunnally, M. Lewis, N. Chakraborty, and K. Sycara, "Levels of automation for human influence of robot swarms," in Proc. Human Factors Ergonomics Soc. Ann. Meeting, 2013, pp. 429-433.

[104] N. E. Leonard, D. A. Paley, R. E. Davis, D. M. Fratantoni, F. Lekien, and F. Zhang, "Coordinated control of an underwater glider fleet in an adaptive ocean sampling field experiment in Monterey bay," J. Field Robot., vol. 27, no. 6, pp. 718-740, 2010.

[105] Z. Kira and M. Potter, "Exerting human control over decentralized robot swarms," in Proc. 4th Int. Conf. Auton. Robot. Agents, 2009, pp. 566-571.

[106] H. Hexmoor, B. McLaughlan, and M. Baker, "Swarm control in unmanned aerial vehicles," in Proc. Int. Conf. Artif. Intell., 2005, pp. 911-917.

[107] H. Hexmoor, S. Eluru, and H. Sabaa, "Plan sharing: Showcasing coordinated UAV formation flight," INFORMATICA, vol. 30, no. 2, p. 183, 2006.

[108] D. S. Brown and M. A. Goodrich, "Human-swarm interactions based on managing attractors," in Proc. ACM/IEEE Int. Conf. Human-Robot Interaction, 2014, pp. 90-97.

[109] A. Steinfeld, O. C. Jenkins, and B. Scassellati, "The oz of wizard: Simulating the human for interaction research," in Proc. 4th ACM/IEEE Int. Conf. Human-Robot Interaction, 2009, pp. 101-107.

[110] M. Daily, Y. Cho, K. Martin, and D. Payton, "World embedded interfaces for human-robot interaction," in Proc. 36th Ann. Hawaii Int. Conf. Syst. Sci., 2003, p. 6.

[111] B. Walter, A. Sannier, D. Reiners, and J. Oliver, "UAV swarm control: Calculating digital pheromone fields with the GPU," J. Defense Model. Simulation: Appl. Methodol., Technol., vol. 3, no. 3, pp. 167-176, 2006.

[112] A. Kolling, S. Nunnally, and M. Lewis, "Towards human control of robot swarms," in Proc. 17th Ann. ACM/IEEE Int. Conf. Human-Robot Interaction, 2012, pp. 89-96.

[113] S. Bashyal and G. Venayagamoorthy, "Human swarm interaction for radiation source search and localization," in Proc. Swarm Intell. Symp., 2008, pp. 1-8.

[114] M. Goodrich, B. Pendleton, P. Sujit, and J. Pinto, "Toward human interaction with bio-inspired robot teams," in Proc. IEEE Int. Conf. Syst. Man, Cybern., 2011, pp. 2859-2864.

[115] M. Goodrich, P. Sujit, S. Kerman, B. Pendleton, and J. Pinto, "Enabling human interaction with bio-inspired robot teams: Topologies, leaders, predators, and stakeholders," Brigham Young Univ., Provo, UT, USA, BYU-HCMI Tech. Rep. 2011-1, 2011.

[116] M. A. Goodrich, S. Kerman, and S.-Y. Jung, "On leadership and influence in human-swarm interaction," in Proc. AAAI Fall Symp. Series. (2012). [Online]. Available: http://www.aaai.org/ocs/index.php/ FSS/FSS12/paper/view/5559

[117] B. Pendleton and M. Goodrich, Scalable Human Interaction with Robotic Swarms. American Institute of Aeronautics and Astronautics, 2015/10/05. (2013). [Online]. Available: http://dx.doi.org/ $10.2514 / 6.2013-4731$

[118] P. Walker, S. A. Amraii, M. Lewis, N. Chakraborty, and K. Sycara, "Human control of leader-based swarms," in Proc. IEEE Int. Conf. Syst. Man, Cybern., 2013, pp. 2712-2717.

[119] P. Walker, S. Amirpour Amraii, M. Lewis, N. Chakraborty, and K. Sycara, "Control of swarms with multiple leader agents," in Proc. IEEE Int. Conf. Syst. Man Cybern., 2014, pp. 3567-3572.

[120] P. Walker, S. Amirpour Amraii, N. Chakraborty, M. Lewis, and K. Sycara, "Human control of robot swarms with dynamic leaders," in Proc. IEEE/RSJ Int. Conf. Intell. Robot. Syst., 2014, pp. 1108-1113.

[121] S. Nunnally, P. Walker, M. Lewis, N. Chakraborty, and K. Sycara, "Using haptic feedback in human robotic swarms interaction," in Proc. Human Factors Ergonomics Soc. Ann. Meeting, 2013, pp. 1047-1051.

[122] S. Sirouspour, "Modeling and control of cooperative teleoperation systems," IEEE Trans. Robot., vol. 21, no. 6, pp. 1220-1225, Dec. 2005.

[123] E. J. Rodriguez-Seda, J. J. Troy, C. A. Erignac, P. Murray, D. M. Stipanovic, and M. W. Spong, "Bilateral teleoperation of multiple mobile agents: Coordinated motion and collision avoidance," IEEE Trans. Control Syst. Technol., vol. 18, no. 4, pp. 984-992, Jul. 2010. 
[124] A. Franchi, P. Robuffo Giordano, C. Secchi, H. I. Son, and H. H. Bulthoff, "A passivity-based decentralized approach for the bilateral teleoperation of a group of UAVs with switching topology," in Proc. IEEE Int. Conf. Robot. Autom., May 2011, pp. 898-905.

[125] Y. Cheung and J. S. Chung, "Cooperative control of a multi-arm system using semi-autonomous telemanipulation and adaptive impedance," in Proc. Int. Conf. Adv. Robot., Jun. 2009, pp. 1-7.

[126] C. Secchi, A. Franchi, H. Bulthoff, and P. R. Giordano, "Bilateral teleoperation of a group of uavs with communication delays and switching topology," in Proc. IEEE Int. Conf. Robot. Autom., 2012, pp. 4307-4314.

[127] D. Lee, A. Franchi, P. Giordano, H. Son, and H. Bulthoff, "Haptic teleoperation of multiple unmanned aerial vehicles over the internet," in Proc. IEEE Int. Conf. Robot. Autom., 2011, pp. 1341-1347.

[128] A. Franchi, C. Masone, H. Bulthoff, and P. Robuffo Giordano, "Bilateral teleoperation of multiple UAVs with decentralized bearing-only formation control," in Proc. IEEE/RSJ Int. Conf. Intell. Robot. Syst., 2011, pp. 2215-2222.

[129] P. Giordano, A. Franchi, C. Secchi, and H. Bülthoff, "Bilateral teleoperation of groups of UAVs with decentralized connectivity maintenance," presented at the Robot.: Sci. Syst. Conf., Los Angeles, CA, USA, 2011.

[130] A. Couture-Beil, R. Vaughan, and G. Mori, "Selecting and commanding individual robots in a multi-robot system," in Proc. Can. Conf. Comput. Robot Vision, 2010, pp. 159-166.

[131] T. Sheridan and W. Verplank, "Human and computer control of undersea teleoperators," DTIC Document, Tech. Rep., 1978.

[132] V. Riley, "A general model of mixed-initiative human-machine systems," in Proc. Human Factors Ergonomics Soc. Ann. Meeting, 1989, pp. $124-128$.

[133] D. Kaber and M. Endsley, "Out-of-the-loop performance problems and the use of intermediate levels of automation for improved control system functioning and safety," Process Safety Prog., vol. 16, no. 3, pp. 126-131, 1997.

[134] H. Ruff, S. Narayanan, and M. Draper, "Human interaction with levels of automation and decision-aid fidelity in the supervisory control of multiple simulated unmanned air vehicles," Presence: Teleoperators Virtual Environ., vol. 11, no. 4, pp. 335-351, 2002.

[135] G. Coppin and F. Legras, "Autonomy spectrum and performance perception issues in swarm supervisory control," Proc. IEEE, vol. 100, no. 3, pp. 590-603, Mar. 2012.

[136] M. Cummings, "Human supervisory control of swarming networks," in Proc. 2nd Ann. Swarming: Auton. Intell. Netw. Syst. Conf., 2004, pp. 1-9.

[137] D. R. Olsen and M. A. Goodrich, "Metrics for evaluating human-robot interactions," in Proc. PERMIS, vol. 2003, 2003, p. 5.

[138] A. Steinfeld, T. Fong, D. Kaber, M. Lewis, J. Scholtz, A. Schultz, and M. Goodrich, "Common metrics for human-robot interaction," in Proc. 1st ACM SIGCHI/SIGART Conf. Human-Robot Interaction, 2006, pp. 33-40.

[139] S. Mau and J. Dolan, "Scheduling for humans in multirobot supervisory control," in Proc. IEEE/RSJ Int. Conf. Intell. Robot. Syst., 2007, pp. $1637-1643$.

[140] S. Nagavalli, L. Luo, N. Chakraborty, and K. Sycara, "Neglect benevolence in human control of robotic swarms," in Proc. IEEE Int. Conf. Robot. Autom., 2014, pp. 6047-6053.

[141] C. E. Harriott, A. E. Seiffert, S. T. Hayes, and J. A. Adams, "Biologicallyinspired human-swarm interaction metrics," in Proc. Human Factors Ergonomics Soc. Ann. Meeting, 2014, pp. 1471-1475.

[142] M. D. Manning, C. E. Harriott, S. T. Hayes, J. A. Adams, and A. E. Seiffert, "Heuristic evaluation of swarm metrics' effectiveness," in Proc. 10th Ann. ACM/IEEE Int. Conf. Human-Robot Interaction Extended Abstr. 2015, pp. 17-18.

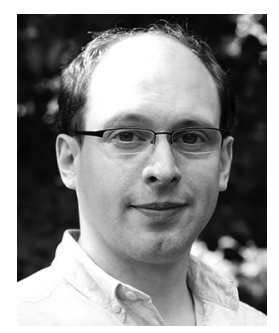

Andreas Kolling (M'10) received the B.S. degree in mathematics and the M.S. degree in computer science from Jacobs University Bremen, Bremen, Germany, in 2004 and 2006, respectively, and the Ph.D. degree in electrical engineering and computer science from the University of California, Merced, CA USA, in 2009.

He is currently a Lecturer with the Department of Automatic Control and Systems Engineering, The University of Sheffield, Sheffield, U.K. His research interests include planning for multirobot systems, pursuit-evasion, graph theory, human-robot interaction, and cooperative robotics.

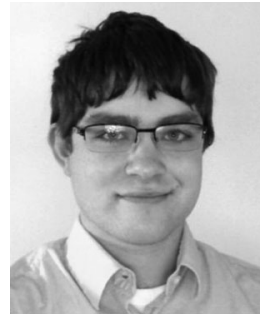

Phillip Walker (S'11) received the B.A. degree in economics and cognitive science from the College of William and Mary, Williamsburg, VA, USA, in 2010, and the M.S. degree in intelligent systems from the University of Pittsburgh, Pittsburgh, PA, USA, in 2013. He is currently working toward the Ph.D. degree in intelligent systems with the University of Pittsburgh.

As a Member of the Usability Lab under the direction of Dr. M. Lewis, his research interests are in human control of robotic swarms, perception of swarm behavior, swarm control algorithms, and artificial intelligence in swarms.

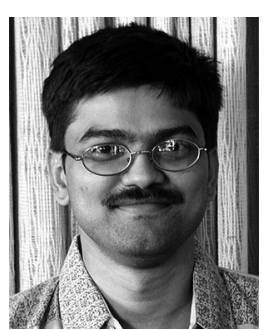

Nilanjan Chakraborty (M'05) received the B.E. degree in mechanical engineering from Jalpaiguri Government Engineering College, Jalpaiguri, India, the M.Sc.(Engg.) degree from the Department of Mechanical Engineering, Indian Institute of Science, Bangalore, India, and the Ph.D. degree from the Department of Computer Science, Rensselaer Polytechnic Institute, Troy, NY, USA.

He is currently an Assistant Professor with the Department of Mechanical Engineering, Stony Brook University, Stony Brook, NY, USA. His research interests include distributed coordination for multiagent systems, human-swarm interaction, and model-predictive motion planning and control for robotic systems.

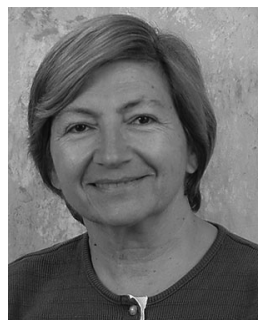

Katia Sycara (F'06) received the B.S. degree in applied mathematics from Brown University, Providence, RI, USA, the M.S. degree in electrical engineering from the University of Wisconsin, Madison, WI, USA, and the Ph.D. degree in computer science from the Georgia Institute of Technology, Atlanta, GA, USA. She holds an Honorary Doctorate from the University of the Aegean, Lesvos, Greece.

She is currently a Professor with the Robotics Institute, School of Computer Science, Carnegie Mellon University, Pittsburgh, PA, USA, and the Director of the Laboratory for Advanced Robotics and Semantic Web Technologies. She has authored more than 600 technical papers dealing with multiagent and multirobot systems, human-robot interaction, complex systems, negotiation, game theory, and data mining.

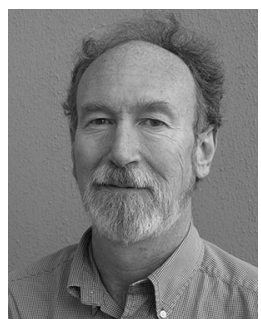

Michael Lewis (M'80) received the Ph.D. degree in engineering psychology from the Georgia Institute of Technology, Atlanta, in 1986

$\mathrm{He}$ is currently a Professor of information sciences and intelligent systems with the University of Pittsburgh, Pittsburgh, PA, USA. His research focuses on human interaction with intelligent automation. He has studied visualization-based control interfaces, human-agent teamwork, and most recently humanrobot interaction. His group with the University of Pittsburgh has developed a number of widely used research tools including USARSim, a robotic simulation adopted for RoboCup competition and, CaveUT, software for inexpensive immersive displays. 